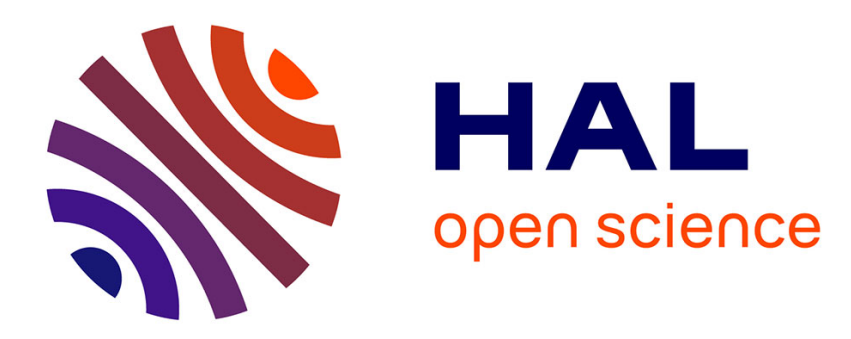

\title{
A DFT study on a calix[5]crown-based heteroditopic receptor
}

Claudia Gargiulli, Giuseppe Gattuso, Anna Notti, Melchiorre F. Parisi, Sebastiano Pappalardo

\section{- To cite this version:}

Claudia Gargiulli, Giuseppe Gattuso, Anna Notti, Melchiorre F. Parisi, Sebastiano Pappalardo. A DFT study on a calix[5]crown-based heteroditopic receptor. Supramolecular Chemistry, 2010, 22 (06), pp.358-364. 10.1080/10610271003678529 . hal-00590751

\section{HAL Id: hal-00590751 https://hal.science/hal-00590751}

Submitted on 5 May 2011

HAL is a multi-disciplinary open access archive for the deposit and dissemination of scientific research documents, whether they are published or not. The documents may come from teaching and research institutions in France or abroad, or from public or private research centers.
L'archive ouverte pluridisciplinaire HAL, est destinée au dépôt et à la diffusion de documents scientifiques de niveau recherche, publiés ou non, émanant des établissements d'enseignement et de recherche français ou étrangers, des laboratoires publics ou privés. 


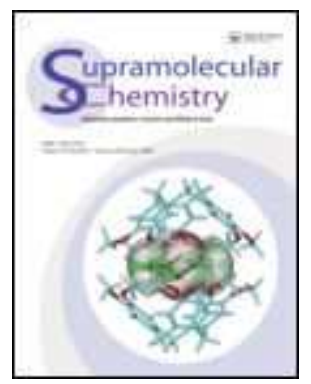

\section{A DFT study on a calix[5]crown-based heteroditopic receptor}

\begin{tabular}{|r|l|}
\hline Journal: & Supramolecular Chemistry \\
\hline Manuscript ID: & GSCH-2009-0131 \\
\hline Manuscript Type: & Full Paper \\
\hline Dute Submitted by the & $19-$ Nov-2009 \\
\hline Complete List of Authors: & $\begin{array}{l}\text { Gargiulli, Claudia; Università di Messina, Dipartimento di Chimica } \\
\text { Organica e Biologica } \\
\text { Gattuso, Giuseppe; Università di Messina, Dipartimento di Chimica } \\
\text { Organica e Biologica } \\
\text { Notti, Anna; Università di Messina, Dipartimento di Chimica } \\
\text { Organica e Biologica } \\
\text { Parisi, Melchiorre; Università di Messina, Dipartimento di Chimica } \\
\text { Organica e Biologica } \\
\text { Pappalardo, Sebastiano; Università di Catania, Dipartimento di } \\
\text { Scienze Chimiche }\end{array}$ \\
\hline Keywords: & $\begin{array}{l}\text { calixarene, alkylammonium ions, ion-pair complexation, } \\
\text { heteroditopic receptor, DFT }\end{array}$ \\
\hline
\end{tabular}

\section{今 scholarONE" \\ Manuscript Central}




1
2
3
4
5
6
7
8
9
10
11
12
13
14
15
16
17
18
19
20
21
22
23
24
25
26
27
28
29
30
31
32
33
34
35
36
37
38
39
40
41
42
43
44
45
46
47
48
49
50
51
52
53
54
55
56
57
58
60
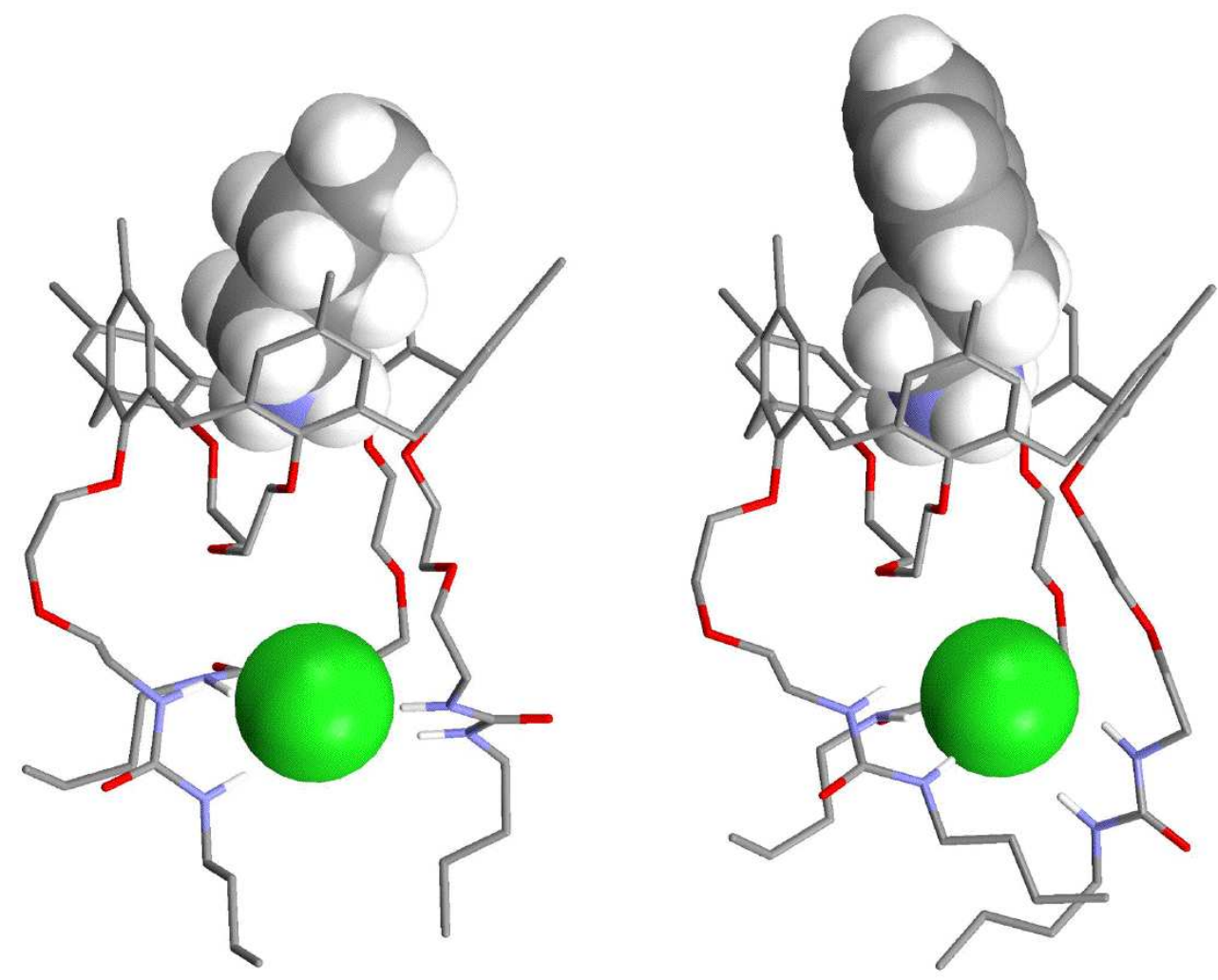

$95 \times 76 \mathrm{~mm}(300 \times 300$ DPI $)$ 


\title{
Supramolecular Chemistry
}

\section{A DFT study on a calix[5]crown-based heteroditopic receptor}

\author{
Claudia Gargiulli ${ }^{\mathrm{a}}$, Giuseppe Gattuso ${ }^{\mathrm{a}}$, Anna Notti ${ }^{\mathrm{a}}$, \\ Sebastiano Pappalardo ${ }^{\mathrm{b}}$ and Melchiorre F. Parisi ${ }^{\mathrm{a}}$ \\ ${ }^{a}$ Dipartimento di Chimica Organica e Biologica, Università di Messina, salita \\ Sperone 31, 98166 Messina, Italy; \\ ${ }^{b}$ Dipartimento di Scienze Chimiche, Università di Catania, Viale A. Doria 6, 95125 \\ Catania, Italy \\ * Corresponding author.
}

Dr. Claudia Gargiulli

Dipartimento di Chimica Organica e Biologica, Università di Messina, salita Sperone 31, 98166 Messina, Italy, Tel. ++39 090 6765177, Email: cgargiulli@ unime.it

Dr. Giuseppe Gattuso

Dipartimento di Chimica Organica e Biologica, Università di Messina, salita Sperone 31, 98166 Messina, Italy, Tel. ++39 090 6765241, Fax ++39 090 392840, Email: gg@isengard.unime.it

Dr. Anna Notti

Dipartimento di Chimica Organica e Biologica, Università di Messina, salita Sperone 31, 98166 Messina, Italy, Tel. ++39 090 6765242, Email: anotti@isengard.unime.it

Prof. Sebastiano Pappalardo

Dipartimento di Scienze Chimiche, Università di Catania, Viale A. Doria 6, 95125 Catania, Italy, Tel. ++39 095 7385018, Email: spappalardo@unict.it

Prof. Melchiorre F. Parisi

Dipartimento di Chimica Organica e Biologica, Università di Messina, salita Sperone 31, 98166 Messina, Italy, Tel. ++39090 6765242, Email: mparisi@ unime.it 


\section{Keywords}

calixarene; alkylammonium ions; ion-pair complexation; heteroditopic receptor; DFT

\section{Introduction}

Complexation of charged substrates by means of neutral receptors was one of the earliest goals of supramolecular chemistry, and considerable effort is still being put into the development of new or improved host molecules for the efficient and selective recognition of both cationic and anionic guests (1). For many years the complexation of these species has been investigated disregarding the role played by the counterion of the target ionic substrate, implicitly circumventing the influence of ion-pairing effects (2) on the strength and selectivity of complexation by employing the so-called 'non-coordinating' counterions (3).

The counterion, however, does indeed play a determinant role, acting in many ways as a competing receptor for the substrate (4). Recently, a new approach has been developed, reliant on the use of hetero(poly)topic receptors, capable of simultaneously - and, allegedly, cooperatively recognising and sequestering both the cationic and the anionic counterparts of a given salt species (5).

Heteroditopic receptors have often been described as more efficient than their parent monotopic receptors, on the basis of transport and/or binding experiments. In many of these examples, efficiency and cooperativity have been deduced by fitting data as 1:1 complexation events. In a very recent paper, however, Roelens and coworkers (6) raised some fundamental questions on the applicability of a 1:1 association model to the interpretation of binding data relative to the complexation of ionic species, demonstrating that ion-pairing equilibria cannot be ignored even in the presence of non-coordinating counterions. In a following report, the same Authors showed that, upon binding of a salt species to a heteroditopic receptor, the 'cooperativity principle is neither general nor predictable' (7).

Over the past few years, we have been actively involved in the design, synthesis and study of heteroditopic (8) and -tetratopic (9) receptors, along with 'binary host' systems based on calix[4](10) or calix[5]arenes (11) for the binding of ion-pairs. In an attempt to verify whether or not 
theoretical methods - which have become a powerful tool in supramolecular chemistry (12) - are able to predict cooperativity effects in the binding of organic salts (13), we have undertaken a study on our most recent heteroditopic receptor, 5,11,17,23,29-pentakis(methyl)-31,33,34-tris-(2-(2- $N$ ( $N$ '-butylureido)ethoxy)ethoxy)-32,35-crown-3-calix[5]arene (1) $(8 a)$, and its affinity toward $n$ butylammonium chloride $(\mathbf{2} \cdot \mathrm{HCl})$ or 2-phenylethylammonium chloride $(\mathbf{3} \cdot \mathrm{HCl})$.

$<$ Block of Structures $>$

\section{Results and discussion}

Based on previous studies by Choe and coworkers (14), who have extensively investigated the binding of alkylammonium ions (15) to monotopic calix[5]arene receptors, we decided to employ a density functional B3LYP/6-311++G(d,p)//B3LYP/6-31G(d) protocol.

The molecular geometries of complexes formed by calix[5]crown $\mathbf{1}$ with (aryl)alkylammonium cations $\mathbf{2} \cdot \mathrm{H}^{+}$and $\mathbf{3} \cdot \mathrm{H}^{+}, \mathrm{Cl}^{-}$ion, as well as $\mathbf{2} \cdot \mathrm{HCl}$ and $\mathbf{3} \cdot \mathrm{HCl}$ salts were initially optimised at the PM3 level in vacuum, and the conformations obtained were then used as a starting point for a full unconstrained reoptimisation at the B3LYP/6-31G(d) level of theory (Figure 1). Single-point energy calculations were then carried out at the B3LYP/6-311++G(d,p) level, to add polarisation functions for hydrogen atoms and diffuse functions for both hydrogen and heavy (non-hydrogen) atoms, in order to gain a more accurate picture of the hydrogen bonding interactions involved in the complexation of the anionic guest (16). In addition, single-point energy calculations were also carried out for the conformations (depicted in cartoon fashion in Figure 2) of the host molecule, the host/guest complexes and the host (and host/guest) structures obtained upon progressive in silico removal of the guest(s). All calculations were carried out in vacuum and results are summarised in Table S1 (see Supporting Information).

$<$ Figure $1>$

$<$ Figure 2>

Calixcrown (17) receptor 1 is capable of simultaneously binding an arylalkyl- or alkylammonium cation and its chloride counterion. The (aryl)alkylammonium guest penetrates the $\pi$-rich aromatic cavity of $\mathbf{1}$, where it is tightly held by ${ }^{+} \mathrm{NH} \cdots \mathrm{O}$ hydrogen bonds and $\mathrm{CH}-\pi$ interactions (18). At the same time, the chloride anion binds to the pocket generated by the ureido hydrogen atoms $(8 a)$. 
In all calculated structures, calix[5]crown 1 was found to adopt a distorted $C_{\mathrm{s}}$ cone-out conformation (19), with one of the aryl groups bearing the crown-3 chain (ring B) leaning outwards with respect to the mean plane generated by the bridging methylene units, and the isolated aryl ring A tilted towards the interior of the cavity (Figure 3). The tilting of the latter ring is much more pronounced in the 'free' receptor $\mathbf{1}$

\section{$<$ Figure 3>}

Despite this self-filling effect, calixarene 1 turns out to be a highly preorganized molecule for the complexation of organic cations. In the presence of an (aryl)alkylammonium ion the cavity of 1 needs to widen only marginally to accommodate the incoming guest and to bring its ammonium group within hydrogen bonding distance of the phenolic oxygen atoms. Rings A and C 'open up' (their tilt angle $\theta$ increases by only $c a .10-20^{\circ}$, vide infra), whereas rings $\mathrm{B}$ and $\mathrm{E}$, the ones connected by the crown-3 ethereal bridge, do not significantly modify their orientation.

This type of guest-induced motion was also observed in the ${ }^{1} \mathrm{H}$ NMR spectra of such complexes in $\mathrm{CDCl}_{3}$. A comparison of the chemical shifts of the ArH singlet in the different host/guest complexes indicates that the deeper the aryl group A is inserted into the cavity, the higher its upfield shift, as a result of an increasing shielding effect induced by the remaining aryl rings (Table 1) (20).

$<$ Table 1>

Figure 3 provides a graphical summary of the calculated conformational changes the calixarene cavity undergoes upon cation, anion or ion pair complexation. The conformational parameters $\phi$ and $\chi$ (21), which are defined as the dihedral angles generated by $\mathrm{C}(1)-\mathrm{C}(2)-\mathrm{C}(3)-\mathrm{C}(4)$ and $\mathrm{C}(2)-\mathrm{C}(3)-\mathrm{C}(4)$ $\mathrm{C}(5)$, respectively, present the $+/-$ alternating sequence typical of a cone conformation and their values, being overall quite similar, indicate comparable structural features. The tilt angles $(\theta)$ of the aryl groups, measured with respect to the mean plane generated by the bridging methylene carbon atoms, are convenient descriptors of the overall conformation assumed by the calixarene cavity.

(Aryl)alkyammonium cations $\mathbf{2} \cdot \mathrm{H}^{+}$and $\mathbf{3} \cdot \mathrm{H}^{+}$sit inside the calixarene cavity, held in place mainly by a single (or two, in the case of $\left[3 \cdot \mathrm{H}^{+} \subset \mathbf{1} \supset \mathrm{Cl}^{-}\right]$) hydrogen bond to the phenolic oxygen atom(s) $(\mathrm{NH} \cdots \mathrm{O}=1.9 \pm 0.1 \AA$, see Figure $\mathrm{S} 1)$. The inability of the ammonium moiety to form tripodal hydrogen bonding is due to the presence of the crown-3 bridge. The latter adopts in all structures a trans-gauche conformation, closing the bottom of the cavity, and thus preventing the (aryl)alkylammonium ion from reaching a more favourable position. In this regard, solid state (18), 
DFT B3LYP/6-31G(d.p) (14c) and HF/6-31G data (14d) on different calix[5]arenes show that the nitrogen ammonium atom must be roughly 1.0-1.1 $\AA$ above the mean plane generated by the phenolic oxygen atoms to be able to fully interact with them. In the present cases, the nitrogen atom lies $2.1 \pm 0.1 \AA$ above that plane.

The ureido-bearing substituents show, as expected, a much higher degree of disorder, their spatial arrangement mainly being driven by $\mathrm{C}=\mathrm{O} \cdots \mathrm{HN}$ hydrogen bonds. However, some common features keep recurring in all the geometry-optimised structures. The free calixarene $\mathbf{1}$ presents, in its minimum energy conformation, an intramolecular hydrogen bond between a ureido $\mathrm{NH}$ hydrogen atom (belonging to the pendant chain attached to ring A) and the central ethereal oxygen atom of the bridging chain $(\mathrm{NH} \cdots \mathrm{O}=2.0 \AA)(22)$. This hydrogen bonding interaction is not lost upon complexation with either of the two cationic guests $\left(\mathrm{NH} \cdots \mathrm{O}=2.1 \AA\right.$ in both $\left[\mathbf{2} \cdot \mathrm{H}^{+} \subset \mathbf{1}\right]$ and $\left.\left[\mathbf{3} \cdot \mathrm{H}^{+} \subset \mathbf{1}\right]\right)$, as the ethereal oxygen atom does not participate in the complexation of the (aryl)alkylammonium ions.

Inclusion of either $\mathbf{2} \cdot \mathrm{H}^{+}$or $\mathbf{3} \cdot \mathrm{H}^{+}$induces a widening of the calixarene cavity, but does not substantially influence the conformational freedom of the lower rim substituents. Even the complexation of chloride ions does not preclude the formation of a weaker hydrogen bond, although in $\left[\mathbf{1} \supset \mathrm{Cl}^{-}\right],\left[\mathbf{2} \cdot \mathrm{H}^{+} \subset \mathbf{1} \supset \mathrm{Cl}^{-}\right]$and $\left[\mathbf{3} \cdot \mathrm{H}^{+} \subset \mathbf{1} \supset \mathrm{Cl}^{-}\right]$the $\mathrm{NH} \cdots \mathrm{O}$ distances are somewhat longer $(2.7 \pm 0.3 \AA)$. Interestingly, in the ion-pair complexes $\left[\mathbf{2} \cdot \mathrm{H}^{+} \subset \mathbf{l} \supset \mathrm{Cl}^{-}\right]$and $\left[3 \cdot \mathrm{H}^{+} \subset \mathbf{1} \supset \mathrm{Cl}^{-}\right]$this ureido hydrogen atom forms a bifurcated hydrogen bond, which also involves the chloride ion $\left(\mathrm{NH} \cdots \mathrm{Cl}^{-}=2.8 \pm 0.1 \AA\right)$. All the remaining ureido hydrogen atoms are involved in $\mathrm{NH} \cdots \mathrm{Cl}^{-}$hydrogen bonds $(2.5 \pm 0.1 \AA)$.

Further insight into the binding behaviour of $\mathbf{1}$ was gained by conveniently analysing the energies of binding, preorganisation and complexation, determined according to the following equations (25):

$$
\begin{aligned}
& \Delta \mathrm{E}_{\text {binding }}=\mathrm{E}_{(\mathrm{HG})}-\mathrm{E}_{(\mathrm{H})}-\mathrm{E}_{(\mathrm{G})} \\
& \Delta \mathrm{E}_{\text {preorg }(\mathrm{H})}=\mathrm{E}_{\text {complexed }(\mathrm{H})}-\mathrm{E}_{(\mathrm{H})} \\
& \Delta \mathrm{E}_{\text {preorg }(\mathrm{G})}=\mathrm{E}_{\text {complexed }(\mathrm{G})}-\mathrm{E}_{(\mathrm{H})} \\
& \Delta \mathrm{E}_{\text {compl }}=\mathrm{E}_{(\mathrm{HG})}-\mathrm{E}_{\text {complexed }(\mathrm{H})}-\mathrm{E}_{\text {complexed }(\mathrm{G})} \\
& \Delta \mathrm{E}_{\text {binding }}=\Delta \mathrm{E}_{\text {compl }}+\Delta \mathrm{E}_{\text {preorg }(\mathrm{H})}+\Delta \mathrm{E}_{\text {preorg(G) }}
\end{aligned}
$$


Complexation of the salt species by calix[5]crown 1 can be dissected into a series of discrete events: (i) conformational rearrangement of $\mathbf{1}$ to accommodate the first ion (either the cation or the anion); (ii) conformational rearrangement of the first ion; (iii) positioning of the first ion into its binding site; (iv) conformational rearrangement of the resulting 1:1 complex to preorganise the second binding site for the incoming counterion; (v) conformational rearrangement of the counterion and (vi) positioning of the counterion into the second binding site.

According to this type of fragmented contribution analysis, data in Table 2 suggest that binding of 2. $\mathrm{HCl}$ and $\mathbf{3} \cdot \mathrm{HCl}$ to 1 takes place with a positive cooperativity. In all instances, binding of a given ion to the free receptor $\mathbf{1}$ is energetically less favoured than that of the same ion to the 1:1 complex (e.g. $\Delta \mathrm{E}_{\text {binding }}=-31.1$ and $-69.7 \mathrm{kcal} / \mathrm{mol}$ for $\mathbf{1}+\mathbf{2} \cdot \mathrm{H}^{+} \rightarrow\left[\mathbf{2} \cdot \mathrm{H}^{+} \subset \mathbf{1}\right]$ and $\left[\mathbf{1} \supset \mathrm{Cl}^{-}\right]+\mathbf{2} \cdot \mathrm{H}^{+} \rightarrow\left[\mathbf{2} \cdot \mathrm{H}^{+} \subset \mathbf{1} \supset \mathrm{Cl}^{-}\right]$, respectively). Similarly, a comparison between the binding energies calculated for either of the two salts and those obtained from the sum of the single ions forming that salt $\left(\Delta \Delta \mathrm{E}_{\text {binding }}=-38.6\right.$ and $37.8 \mathrm{kcal} / \mathrm{mol}$ for $2 \cdot \mathrm{HCl}$ and $3 \cdot \mathrm{HCl}$, respectively) (26) reveals that ion-pair complexation is significantly favoured, and proceeds with a stabilisation greater than that obtained from the mere sum of the contributions of the two 1:1 binding events. These figures can be explained by taking into account the stabilising contribution provided by the electrostatic attraction between the two ions included in the binding sites of the heteroditopic receptor $\left({ }^{+} \mathrm{N} \cdots \mathrm{Cl}^{-}=7.3\right.$ and $7.0 \AA$ for $\left[\mathbf{2} \cdot \mathrm{H}^{+} \subset \mathbf{1} \supset \mathrm{Cl}^{-}\right]$ and $\left[\mathbf{3} \cdot \mathrm{H}^{+} \subset \mathbf{1} \supset \mathrm{Cl}^{-}\right]$, respectively).

As far as the conformational rearrangement of the host is concerned, different trends are observed upon sequential ion binding. The first binding event proceeds with similar preorganisation energies for both anion and cations $\left(\Delta \mathrm{E}_{\text {preorg }(\mathrm{H})}=7.3-9.4 \mathrm{kcal} / \mathrm{mol}\right)$, indicating that comparable energy penalties are paid for the 'opening' of the cavity and the 'unraveling' of the ureido-bearing chains. Once the anionic complex $\left(\left[1 \supset \mathrm{Cl}^{-}\right]\right)$is formed, preorganisation of the calixarene cavity to receive either of the two incoming cations $\left(\mathbf{2} \cdot \mathrm{H}^{+}\right.$or $\left.\mathbf{3} \cdot \mathrm{H}^{+}\right)$requires an energy contribution similar to that calculated for the free receptor $\left(\Delta \mathrm{E}_{\text {preorg(H) }}=8.410 .2\right.$ vs $\left.7.3-9.7 \mathrm{kcal} / \mathrm{mol}\right)$. Conversely, preorganisation of the anionic binding site of both cationic complexes $\left(\left[2 \cdot \mathrm{H}^{+} \subset \mathbf{1}\right]\right.$ and $\left.\left[3 \cdot \mathrm{H}^{+} \subset \mathbf{1}\right]\right)$ to host the chloride ion suffers from 


\section{Conclusions}

DFT B3LYP/6-311++G(d,p)//B3LYP/6-31G(d) calculations have shown that ion-pair complexation of $n$-butylammonium or 2-phenylethylammonium chloride to the two binding sites of a calix[5]arene-based receptor takes place with positive cooperativity. Remarkable agreement between experimental ${ }^{1} \mathrm{H}$ NMR and calculated data on the binding geometries of both single ion and salt species to this receptor has also been found. Overall, our findings demonstrate that DFT studies can be used as a valuable tool for predicting the binding energies and structural features of heteroditopic receptors involved in ion-pair complexation of organic salt species.

\section{Experimental}

\section{Molecular Modeling}

The conformational analysis of calix[5]crown $\mathbf{1}$, complexes $\left[\mathbf{1} \supset \mathrm{Cl}^{-}\right],\left[\mathbf{2} \cdot \mathrm{H}^{+} \subset \mathbf{1}\right],\left[\mathbf{2} \cdot \mathrm{H}^{+} \subset \mathbf{1} \supset \mathrm{Cl}^{-}\right]$, $\left[\mathbf{3} \cdot \mathrm{H}^{+} \subset \mathbf{1}\right]$ and $\left[\mathbf{3} \cdot \mathrm{H}^{+} \subset \mathbf{1} \supset \mathrm{Cl}^{-}\right]$was carried out with the classical molecular mechanics force field (MMFF) by using the Monte Carlo method to randomly sample the conformational space. The equilibrium geometries were first refined at the PM3 level, and the resulting conformations were used as input for the calculations at the density functional level of theory (DFT, B3LYP functional) 
using the 6-31G(d) basis set. Single-point energy calculations were performed at the DFT B3LYP/6-311++G(d,p) level of theory. All quantum mechanical calculations were performed using Spartan'08 (Wavefunction, Inc.) on a Macintosh equipped with Intel Dual Quad Core CPUs at 3.2 GHz.

\section{${ }^{1}$ H NMR Experiments}

${ }^{1} \mathrm{H}$ NMR spectra were recorded at room temperature in $\mathrm{CDCl}_{3}$, at $500 \mathrm{MHz}$, using the residual solvent signal as the internal standard. All spectra were recorded on equimolar solutions of host and guest $(1 \mathrm{mM})$. The following stock solutions were used: $[\mathbf{1}]=10 \mathrm{mM}$ in $\mathrm{CDCl}_{3}$; [(aryl)alkylammonium picrate $]=10 \mathrm{mM}$ in $\mathrm{CD}_{3} \mathrm{OD}$. Complexes of calix[5]crown 1 with picrate salts were prepared by adding $70 \mu \mathrm{L}$ of stock solutions of each component, evaporating the solvents to dryness, and redissolving the residue in $\mathrm{CDCl}_{3}$ to a final volume of $700 \mu \mathrm{L}$, for a final concentration of host and guest of $1 \mathrm{mM}$.

\section{Acknowledgments}

We are indebted to Professor G. Bruno (Università di Messina) for some stimulating discussions and for his helpful advice.

\section{Supporting information available}

Additional figures, calculated energies and coordinates for all geometry-optimised structures. 


\section{References}

(1) Steed, J.W.; Atwood, J.L. Cation Binding Hosts, In Supramolecular Chemistry, 2nd ed.; John Wiley \& Sons: Chichester, 2009; pp 105-222; Katayev, E.A.; Melfi, P.J.; Sessler, J.L. Anion Binding Macrocycles, In Modern Supramolecular Chemistry; Diederich, F., Stangg, P.J., Tykwinski, R.R., Eds.; Wiley-VCH: Weinheim, 2008; pp 315-347.

(2) Marcus, Y.; Hefter, G. Chem. Rev. 2006, 106, 4585-4621; Isaacs, N. Physical Organic Chemistry, 2nd ed.; Longmans: England, 1995; pp 56-62.

(3) Krossing, I.; Raabe, I. Angew. Chem. Int. Ed. 2004, 43, 2066-2090.

(4) Gasa, T.B.; Spruell, J.M.; Dichtel, W.R.; Sørensen, T.J.; Philp, D.; Stoddart, J.F.; Kuzmic, P. Chem. Eur. J. 2009, 15, 106-116; Huang, F.; Jones, J.W.; Gibson, H.W. J. Org. Chem. 2007, 72, 6573-6576; Sessler, J.L.; Gross, D.E.; Cho, W.-S.; Lynch, V.M.; Schmidtchen, F.P.; Bates, G.W.; Light, M.E.; Gale, P.A. J. Am. Chem. Soc. 2006, 128, 12281-12288; Huang, F.; Jones, J.W.; Slebodnick, C.; Gibson, H.W. J. Am. Chem. Soc. 2003, 125, 14458-14464; Jones, J.W.; Gibson, H.W. J. Am. Chem. Soc. 2003, 125, 7001-7004.

(5) For recent reviews on ion-pair receptors, see: Sessler, J.L.; Gale, P.A.; Cho, W.-S. In Anion Receptor Chemistry; RSC: Cambridge, 2006; pp 259-293; Smith, B.D. In Macrocyclic Chemistry: Current Trends and Future Perspectives; Gloe K., Ed.; Springer: Dordrecht, The Netherlands, 2005; pp 137-151; Gale, P.A. Coord. Chem. Rev. 2003, 240, 191-221; Beer, P.D.; Gale, P.A. Angew. Chem. Int. Ed. 2001, 40, 487-516. For some selected examples, see: Sessler, J.L.; Kim, S.K.; Gross, D.E.; Lee, C.-H.; Kim, J.S.; Lynch, V.M. J. Am. Chem. Soc. 2008, 130, 13162-13166; Le Gac, S.; Ménand, M.; Jabin, I. Org. Lett. 2008, 10, 5195-5198; Cametti, M.; Nissinen, M.; Dalla Cort, A.; Mandolini, L.; Rissanen, K. J. Am. Chem. Soc. 2007, 129, 3641-3648; Mele, A.; Metrangolo, P.; Neukirch, H.; Pilati T.; Resnati, G. J. Am. Chem. Soc. 2005, 127, 14972-14973; Turner, D.R.; Spencer, E.C.; Howard, J.A.K.; Tocher D.A.; Steed, J.W. Chem. Commun. 2004, 1352-1353; Atwood J.L.; Szumna, A. Chem. Commun. 2003, 940-941; Mahoney, J.M.; Davis, J.P.; Beatty, A.M.; Smith, B.D. J. Org. Chem. 2003, 68, 9819-9820.

(6) Roelens, S.; Vacca, A.; Venturi, C. Chem. Eur. J. 2009, 15, 2635-2644, and references therein.

(7) Roelens, S.; Vacca, A.; Francesconi, O.; Venturi, C. Chem. Eur. J. 2009, 15, 8296-8302.

(8) (a) Gargiulli, C.; Gattuso, G.; Liotta, C.; Notti, A.; Parisi, M.F.; Pisagatti, I.; Pappalardo, S. J. Org. Chem. 2009, 74, 4350-4353; (b) Ballistreri, F.P.; Notti, A.; Pappalardo, S.; Parisi, M.F.; Pisagatti, I. Org. Lett. 2003, 5, 1072-1074.

(9) Garozzo, D.; Gattuso, G.; Notti, A.; Pappalardo, A.; Pappalardo, S.; Parisi, M.F.; Perez, M.; Pisagatti, I. Angew. Chem. Int. Ed. 2005, 45, 4892-4896. 
(10) Gattuso, G.; Pappalardo, A.; Parisi, M.F.; Pisagatti, I.; Crea, F.; Liantonio, R.; Metrangolo, P.; Navarrini, W.; Resnati, G.; Pilati, T.; Pappalardo, S. Tetrahedron 2007, 63, 4951-4958; Gattuso, G.; Liantonio, R.; Meyer, F.; Metrangolo, P.; Resnati, G.; Pappalardo, A.; Parisi, M.F.; Pilati, T.; Pisagatti, I. Supramol. Chem. 2006, 18, 235-243.

(11) Biella, S.; Gattuso, G.; Notti, A.; Metrangolo, P.; Pappalardo, S.; Parisi, M.F.; Pilati, T.; Resnati, G.; Terraneo, G. Supramol. Chem. 2009, 21, 149-156; Cafeo, G.; Gattuso, G.; Kohnke, F.H.; Notti, A; Occhipinti, S.; Pappalardo, S.; Parisi, M.F. Angew. Chem. Int. Ed. 2002, 41, 2122-2126; Cafeo, G.; Gargiulli, C.; Gattuso, G.; Kohnke, F.H.; Notti, A; Occhipinti, S.; Pappalardo, S.; Parisi, M.F. Tetrahedron Lett. 2002, 43, 8103-8106.

(12) Sheehan, R.; Cragg, P.J. Supramol. Chem. 2008, 20, 443-451; Yan, S.; Lee, S.J.; Kang, S.; Lee, J.Y. Supramol. Chem. 2007, 19, 229-241; Schatz, J. Ab initio Calculations on Supramolecular Systems In Encyclopedia of Supramolecular Chemistry; Taylor \& Francis: London, 2006. For reviews on the applications of ab initio quantum chemical calculations on calixarenes, see: Mohammed-Ziegler, I.; Billes, F. J. Incl. Phenom. Macrocycl. Chem. 2007, 58, 19-42; Schatz, J. Collect. Czech. Chem. Commun. 2004, 69, 1169-1194.

(13) For a recent example of MD simulations on calix[4]arene-based heteroditopic receptors, see: Lankshear, M.D.; Dudley, I.M.; Chan, K.-M.; Cowley, A.R.; Santos, S.M.; Felix, V.; Beer, P.D. Chem. Eur. J. 2008, 14, 2248-2263. For a DFT B3LYP/6-31G(d) study on a crownether-based heteroditopic receptor, see: Vivas-Reyes, R.; De Proft, F.; Biesemans, M.; Willem, R.; Geerlings, P. Eur. J. Inorg. Chem. 2003, 1315-1324.

(14) (a) Kim, K.; Lee, S.-H.; Choe, J.-I. Bull Korean Chem. Soc. 2008, 29, 2152-2156; (b) Kim, K.; Park, S.J. Choe, J.-I. Bull Korean Chem. Soc. 2008, 29, 1893-1897; (c) Oh, D.; Choe, J.-I. Bull Korean Chem. Soc. 2007, 28, 596-600; (d) Choe, J.-I.; Lee, S.H.; Oh, D.-S.; Chang, S.K.; Nanbu, S. Bull Korean Chem. Soc. 2004, 25, 190-194, (e) Choe, J.-I.; Chang, S.-K.; Satoshi, M.; Nanbu, S. Bull Korean Chem. Soc. 2003, 24, 75-80; (f) Choe, J.-I.; Chang, S.-K. Bull Korean Chem. Soc. 2002, 23, 48-52.

(15) Binding of $n$-butylammonium salts to a calix[4]arene has been recently investigated, see Kríz, J.; Dybal, J.; Budka, J.; Makrlík, E. Magn. Reson. Chem. 2008, 46, 399-407.

(16) Hehre, W.J.; Radom, L.; Schleyer, P.R.; Pople, J.A. Ab Initio Molecular Orbital Theory; Wiley: New York, 1985.

(17) For recent reviews on calixcrowns, see: Salorinne, K.; Nissinen, M. J. Incl. Phenom. Macrocycl. Chem. 2008, 61, 11-27; Casnati, A.; Ungaro, R.; Asfari, Z.; Vicens, J. in Calixarenes 2001; Asfari, Z., Böhmer, V., Harrowfield, J., Vicens, J., Eds.; Kluwer Academic Publishers: Dordrecht, 2001, pp 365-384. For examples of calix[5]crowns, see: Gattuso, G.; Liotta, C.; Notti, A.; Parisi, M.F.; Pisagatti, I.; Pappalardo, S. Tetrahedron Lett. 2008, 49, 
7146-7148; De Salvo, G.; Gattuso, G.; Notti, A.; Parisi, M.F.; Pappalardo, S. J. Org. Chem. 2002, 67, 684-692.

(18) Gattuso, G.; Notti, A.; Pappalardo, S.; Parisi, M.F.; Pilati, T.; Resnati, G.; Terraneo, G. CrystEngComm 2009 11, 1204-1206.

(19) Stewart, D.R.; Krawiec, M.; Kashyap, R.P.; Watson, W.H.; Gutsche, C.D. J. Am. Chem. Soc. 1995, 117, 586-601. For an exhaustive MM study on calix[5]arene conformations, see: Thondorf, I.; Brenn, J. J. Chem. Soc., Perkin Trans. 2 1997, 2293-2299.

(20) ${ }^{1} \mathrm{H}$ and ${ }^{13} \mathrm{C}$ NMR chemical shifts for some relevant hydrogen and carbon atoms in calixarenes have been correlated to different conformations by GIAO calculations, see: Bifulco, G.; Riccio, R.; Gaeta, C.; Neri, P. Chem. Eur. J. 2007, 13, 7185-7194; Bifulco, G.; GomezPaloma, L.; Riccio, R.; Gaeta, C.; Troisi, F.; Neri, P. Org. Lett. 2005, 7, 5757-5760.

(21) Ugozzoli, F.; Andreetti, G.D. J. Inclusion Phenom. 1992, 13, 337-348.

(22) In a calix[4]arene derivative bearing two ureido groups at its lower rim, semi-empirical calculations at the PM3 level showed a similar folding of the pendant chain as a result of hydrogen bonding. See: Yakovenko, A.V.; Boyko, V.I.; Kalchenko, V.I.; Baldini, L.; Casnati, A.; Sansone, F.; Ungaro, R. J. Org. Chem. 2007, 72, 3223-3231.

(23) With the exception of $\left[1 \supset \mathrm{Cl}^{-}\right] n-\mathrm{Bu}_{4} \mathrm{~N}^{+}$, all complexes give rise to slow exchange on the NMR time scale, providing direct observation of both complexed and free species. See: ArnaudNeu, F.; Fuangswasdi, S.; Notti, A.; Pappalardo, S.; Parisi, M.F. Angew. Chem. Int. Ed. 1998, 37, 112-114.

(24) Neither picrate nor tetra- $n$-butylammonium ions bind to calix[5]crown $\mathbf{1}$. They were therefore used as non-coordinating counterions in order to gather structural information on the 1:1 complexes $\left(\left[\mathbf{1} \supset \mathrm{Cl}^{-}\right],\left[\mathbf{2} \cdot \mathrm{H}^{+} \subset \mathbf{1}\right]\right.$ and $\left.\left[\mathbf{3} \cdot \mathrm{H}^{+} \subset \mathbf{1}\right]\right)$ by ${ }^{1} \mathrm{H} \mathrm{NMR}$.

(25) Ruangpornvisuti, V. J. Mol. Struct. (Teochem) 2004, 686, 47-55. See also: Marcos, P.M.; Ascenso, J.R.; Segurado, M.A.P.; Bernardino, R.J.; Cragg, P.J. Tetrahedron 2009, 65, 496503.

(26) $\Delta \Delta \mathrm{E}_{\text {binding }}$ is defined as follows: $\Delta \Delta \mathrm{E}_{\text {binding }}[\mathbf{2} \cdot \mathrm{HCl}]=\Delta \mathrm{E}_{\text {binding }}\left[\mathbf{2} \cdot \mathrm{H}^{+} \subset \mathbf{1} \supset \mathrm{Cl}^{-}\right]-$ $\Delta \mathrm{E}_{\text {binding }}\left[\mathbf{2} \cdot \mathrm{H}^{+} \subset \mathbf{1}\right]-\Delta \mathrm{E}_{\text {binding }}\left[\mathbf{1} \supset \mathrm{Cl}^{-}\right]$for $\mathbf{2} \cdot \mathrm{HCl}$, and $\Delta \Delta \mathrm{E}_{\text {binding }}[\mathbf{3} \cdot \mathrm{HCl}]=\Delta \mathrm{E}_{\text {binding }}\left[\mathbf{3} \cdot \mathrm{H}^{+} \subset \mathbf{1} \supset \mathrm{Cl}^{-}\right]$ $-\Delta \mathrm{E}_{\text {binding }}\left[3 \cdot \mathrm{H}^{+} \subset \mathbf{1}\right]-\Delta \mathrm{E}_{\text {binding }}\left[\mathbf{1} \supset \mathrm{Cl}^{-}\right]$for $\mathbf{3} \cdot \mathrm{HCl}$.

(27) Yatsimirsky, A.K. The Allosteric Effect In Encyclopedia of Supramolecular Chemistry; Taylor \& Francis: London, 2004.

(28) Svorstol, I.; Hoiland, H.; Songstad, J. Acta Chem. Scand. B 1984, 38, 885-893; Balt, S.; du Chattel, G.; de Kieviet, W.; Tellman, A. Z. Naturforsch. Teil. B 1978, 754-749. 
Table 1. Comparison between the tilt angle of ring A and the chemical shift (23) of its aromatic hydrogen atoms $\left(\mathrm{CDCl}_{3}, 1 \mathrm{mM}, 500 \mathrm{MHz}, 25^{\circ} \mathrm{C}\right)$.

\begin{tabular}{lclc}
\hline calculated & tilt angle $(\theta)$ & experimental & ArH $\delta(\mathrm{ppm})$ \\
\hline $\mathbf{1}$ & $69.9^{\circ}$ & $\mathbf{1}$ & 6.17 \\
{$\left[\mathbf{1} \supset \mathrm{Cl}^{-}\right]$} & $74.4^{\circ}$ & {$\left[\mathbf{1} \supset \mathrm{Cl}^{-}\right] n-\mathrm{Bu}_{4} \mathrm{~N}^{+\mathrm{a}}$} & $6.10^{\mathrm{b}}$ \\
{$\left[\mathbf{2} \cdot \mathrm{H}^{+} \subset \mathbf{1}\right]$} & $87.7^{\circ}$ & {$\left[\mathbf{2} \cdot \mathrm{H}^{+} \subset \mathbf{1}\right] \mathrm{Pic}^{-\mathrm{a}}$} & 6.43 \\
{$\left[\mathbf{2} \cdot \mathrm{H}^{+} \subset \mathbf{1} \supset \mathrm{Cl}^{-}\right]$} & $84.8^{\circ}$ & {$\left[\mathbf{2} \cdot \mathrm{H}^{+} \subset \mathbf{1} \supset \mathrm{Cl}^{-}\right]$} & 6.46 \\
{$\left[\mathbf{3} \cdot \mathrm{H}^{+} \subset \mathbf{1}\right]$} & $86.5^{\circ}$ & {$\left[\mathbf{3} \cdot \mathrm{H}^{+} \subset \mathbf{1}\right] \mathrm{Pic}^{-\mathrm{a}}$} & 6.31 \\
{$\left[\mathbf{3} \cdot \mathrm{H}^{+} \subset \mathbf{1} \supset \mathrm{Cl}^{-}\right]$} & $87.6^{\circ}$ & {$\left[\mathbf{3} \cdot \mathrm{H}^{+} \subset \mathbf{1} \supset \mathrm{Cl}^{-}\right]$} & 6.35 \\
\hline
\end{tabular}

${ }^{a}$ Tetra- $n$-butylammonium and picrate $\left(\mathrm{Pic}^{-}\right)$were employed as non-coordinating counterions (24). ${ }^{\mathrm{b}}$ Estimated from Benesi-Hildebrandt linearisation of titration experiments. 
Table 2. DFT B3LYP/6-311++G(d,p)//B3LYP/6-31G(d) calculated binding, complexation and preorganisation energies $(\mathrm{kcal} / \mathrm{mol})$ for the complexes of calix [5]crown $\mathbf{1}$ and $\mathbf{2} \cdot \mathrm{H}^{+}, \mathbf{3} \cdot \mathrm{H}^{+}, \mathrm{Cl}^{-}, \mathbf{2} \cdot \mathrm{HCl}$ and $\mathbf{3} \cdot \mathrm{HCl}$.

\begin{tabular}{lrrrr}
\hline \multicolumn{1}{c}{ Species } & $\Delta \mathrm{E}_{\text {preorg }(\mathrm{H})}$ & $\Delta \mathrm{E}_{\text {preorg }(\mathrm{G})}{ }^{\mathrm{a}}$ & $\Delta \mathrm{E}_{\text {compl }}$ & \multicolumn{1}{c}{$\Delta \mathrm{E}_{\text {binding }}$} \\
\hline $\mathbf{1}+\mathrm{Cl}^{-}$ & 9.4 & - & -62.8 & -53.4 \\
$\mathbf{1}+\mathbf{2} \cdot \mathrm{H}^{+}$ & 9.7 & 0.3 & -41.1 & -31.1 \\
{$\left[\mathbf{1} \supset \mathrm{Cl}^{-}\right]+\mathbf{2} \cdot \mathrm{H}^{+}$} & 10.2 & 1.2 & -81.0 & -69.7 \\
{$\left[\mathbf{2} \cdot \mathrm{H}^{+} \subset \mathbf{1}\right]+\mathrm{Cl}^{-}$} & 17.3 & 0.9 & -109.3 & -91.2 \\
$\mathbf{1}+\mathbf{2} \cdot \mathrm{H}^{+}+\mathrm{Cl}^{-}$ & 27.0 & 1.2 & -151.3 & -123.1 \\
$\mathbf{1}+\mathbf{3} \cdot \mathrm{H}^{+}$ & 7.3 & 0.7 & -38.4 & -30.4 \\
{$\left[\mathbf{1} \supset \mathrm{Cl}^{-}\right]+\mathbf{3} \cdot \mathrm{H}^{+}$} & 8.4 & 0.7 & -77.3 & -68.2 \\
{$\left[\mathbf{3} \cdot \mathrm{H}^{+} \subset \mathbf{1}\right]+\mathrm{Cl}^{-}$} & 15.2 & 0.0 & -106.5 & -91.3 \\
$\mathbf{1}+\mathbf{3} \cdot \mathrm{H}^{+}+\mathrm{Cl}^{-}$ & 20.5 & 0.7 & -142.8 & -121.6 \\
\hline
\end{tabular}

${ }^{\mathrm{a}}$ Relative to the cationic guest, as for $\mathrm{Cl}^{-} \Delta \mathrm{E}_{\text {preorg(G) }}=0$. 


\section{Captions to Figures}

Figure 1. Optimised B3LYP/6-31-G(d) geometries for calix[5]crown $\mathbf{1}$ and its complexes with $\mathbf{2} \cdot \mathrm{H}^{+}$, $\mathbf{3} \cdot \mathrm{H}^{+}, \mathrm{Cl}^{-}, \mathbf{2} \cdot \mathrm{HCl}$ and $\mathbf{3} \cdot \mathrm{HCl}$.

Figure 2. Cartoon representation of the complexes of calix[5]crown $\mathbf{1}$ with the cationic and/or anionic guest(s) described in this paper. Dashed arrows indicate in silico guest removal. $\mathrm{R}=n$ $\mathrm{BuNH}_{3}{ }^{+}\left(\mathbf{2} \cdot \mathrm{H}^{+}\right)$or $\mathrm{PhCH}_{2} \mathrm{CH}_{2} \mathrm{NH}_{3}{ }^{+}\left(\mathbf{3} \cdot \mathrm{H}^{+}\right)$.

Figure 3. Radial plots comparing tilt angles $(\theta)$ and the torsions angles $\phi$ and $\chi$ for free calix[5]crown $\mathbf{1}$ and its complexes with $\mathbf{2} \cdot \mathrm{H}^{+}, \mathbf{3} \cdot \mathrm{H}^{+}, \mathrm{Cl}^{-}, \mathbf{2} \cdot \mathrm{HCl}$ and $\mathbf{3} \cdot \mathrm{HCl}$. 

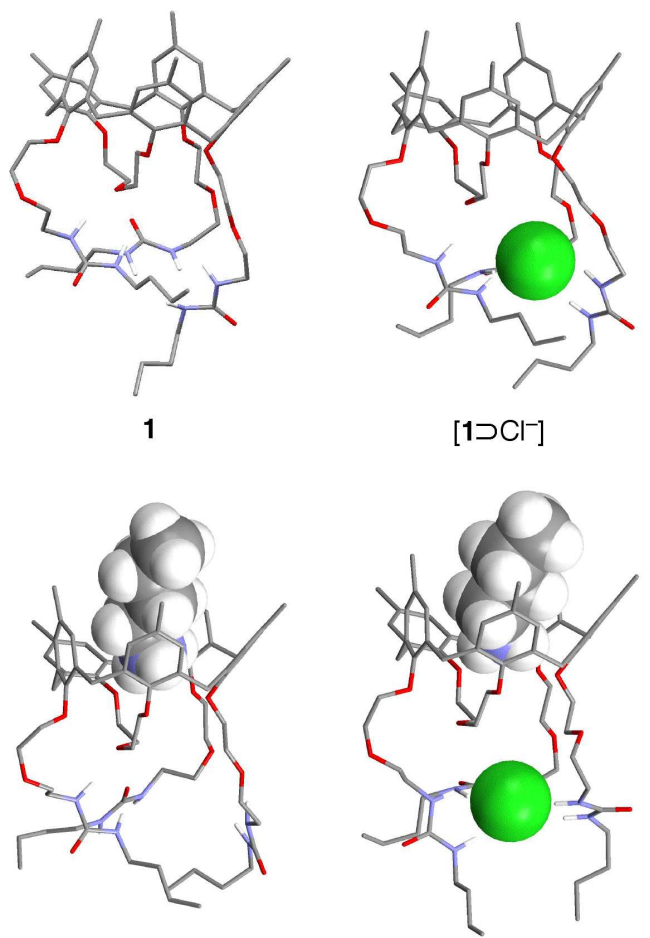

$\left[2 \cdot H^{+} \subset 1\right]$

$\left[2 \cdot \mathrm{H}^{+} \subset 1 \supset \mathrm{Cl}^{-}\right]$
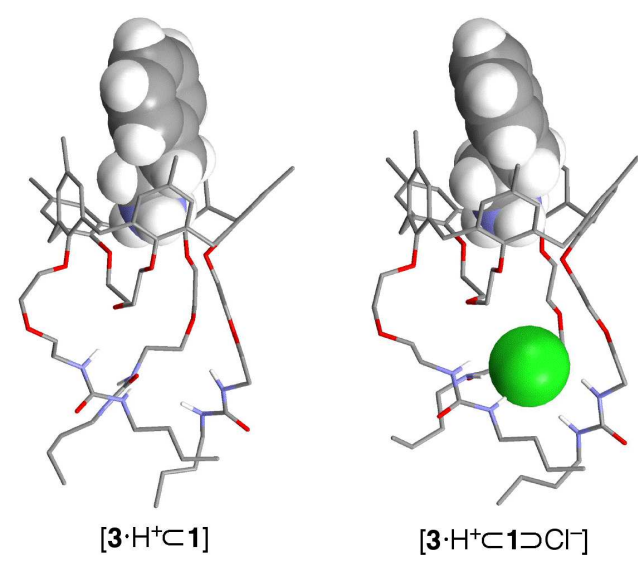

Figure 1. Optimised B3LYP/6-31-G(d) geometries for calix[5]crown $\mathbf{1}$ and its complexes with $\mathbf{2} \cdot \mathrm{H}^{+}$, $\mathbf{3} \cdot \mathrm{H}^{+}, \mathrm{Cl}^{-}, \mathbf{2} \cdot \mathrm{HCl}$ and $\mathbf{3} \cdot \mathrm{HCl}$.

$98 \times 237 \mathrm{~mm}(300 \times 300 \mathrm{DPI})$ 

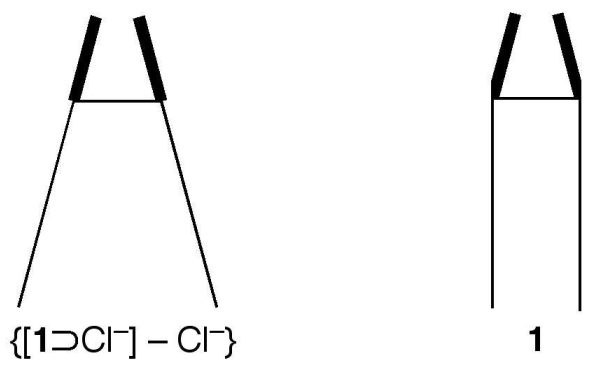

1
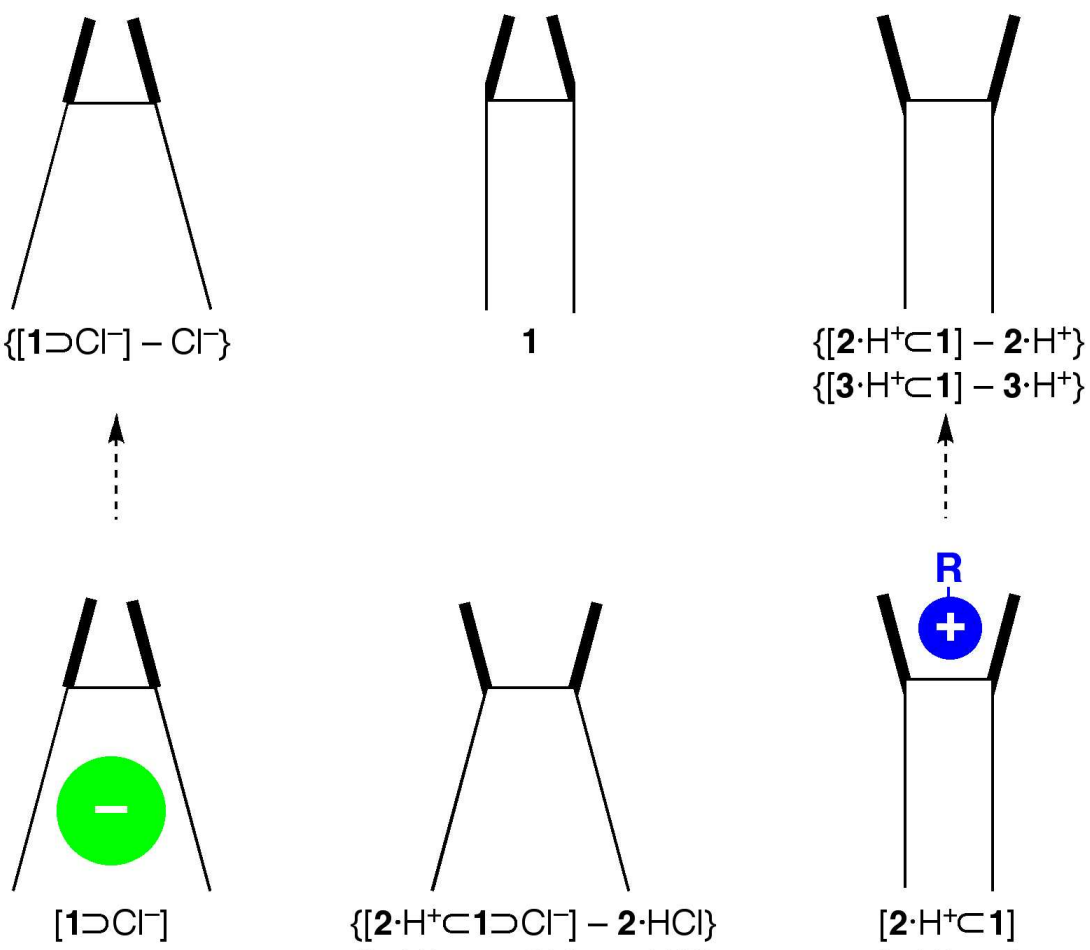
$\left\{\left[3 \cdot \mathrm{H}^{+} \subset 1 \supset \mathrm{Cl}^{-}\right]-3 \cdot \mathrm{HCl}\right\}$

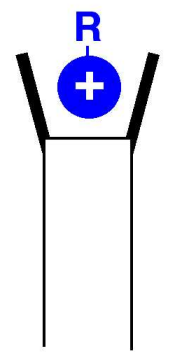

$\left[2 \cdot \mathrm{H}^{+} \subset 1\right]$ $\left[3 \cdot H^{+} \subset 1\right]$

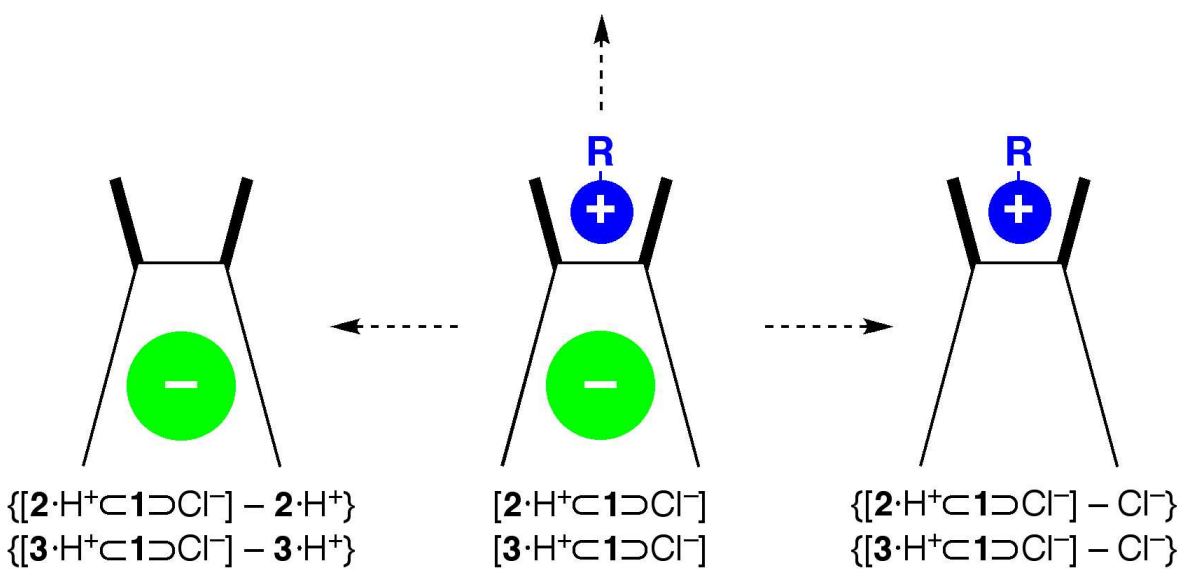

Figure 2. Cartoon representation of the complexes of calix[5]crown $\mathbf{1}$ with the cationic and/or anionic guest(s) described in this paper. Dashed arrows indicate in silico guest removal. $\mathrm{R}=n$ $\mathrm{BuNH}_{3}{ }^{+}\left(\mathbf{2} \cdot \mathrm{H}^{+}\right)$or $\mathrm{PhCH}_{2} \mathrm{CH}_{2} \mathrm{NH}_{3}{ }^{+}\left(\mathbf{3} \cdot \mathrm{H}^{+}\right)$. $142 \times 189 \mathrm{~mm}(300 \times 300 \mathrm{DPI})$ 


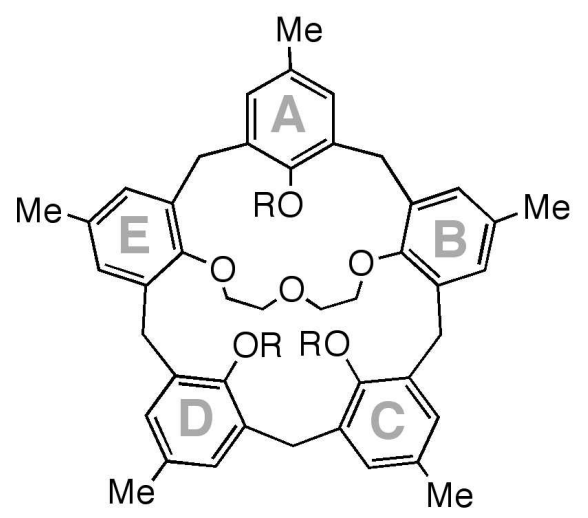<smiles>COc1c(C)cc(C)cc1Cc1cc(C)cc(C)c1OC</smiles>
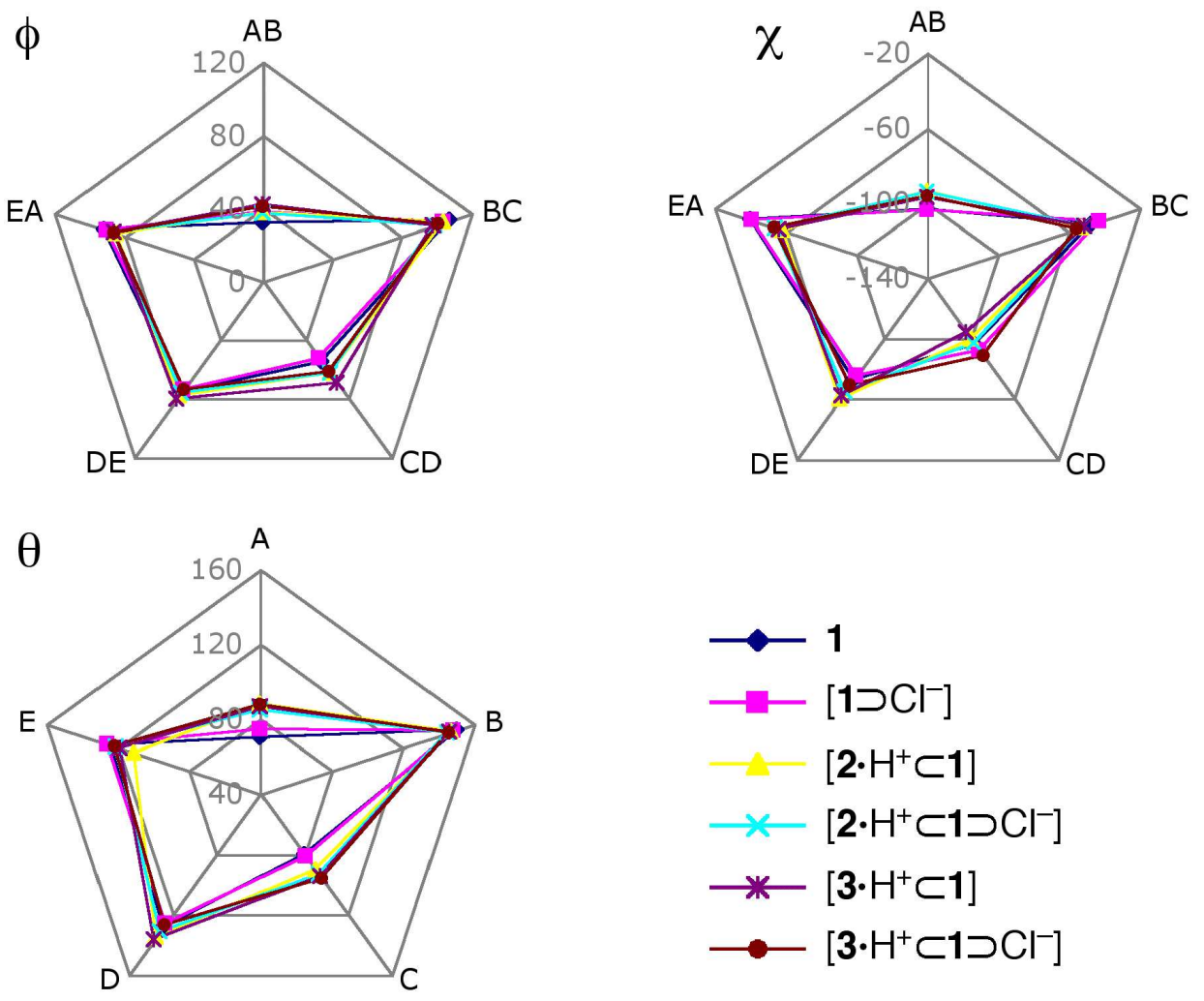

Figure 3. Radial plots comparing tilt angles $(\theta)$ and the torsions angles $\Phi$ and $\mathrm{x}$ for free calix[5]crown 1 and its complexes with $\mathbf{2} \cdot \mathrm{H}^{+}, \mathbf{3} \cdot \mathrm{H}^{+}, \mathrm{Cl}^{-}, \mathbf{2} \cdot \mathrm{HCl}$ and $\mathbf{3} \cdot \mathrm{HCl}$. $153 \times 192 \mathrm{~mm}(300 \times 300 \mathrm{DPI})$ 


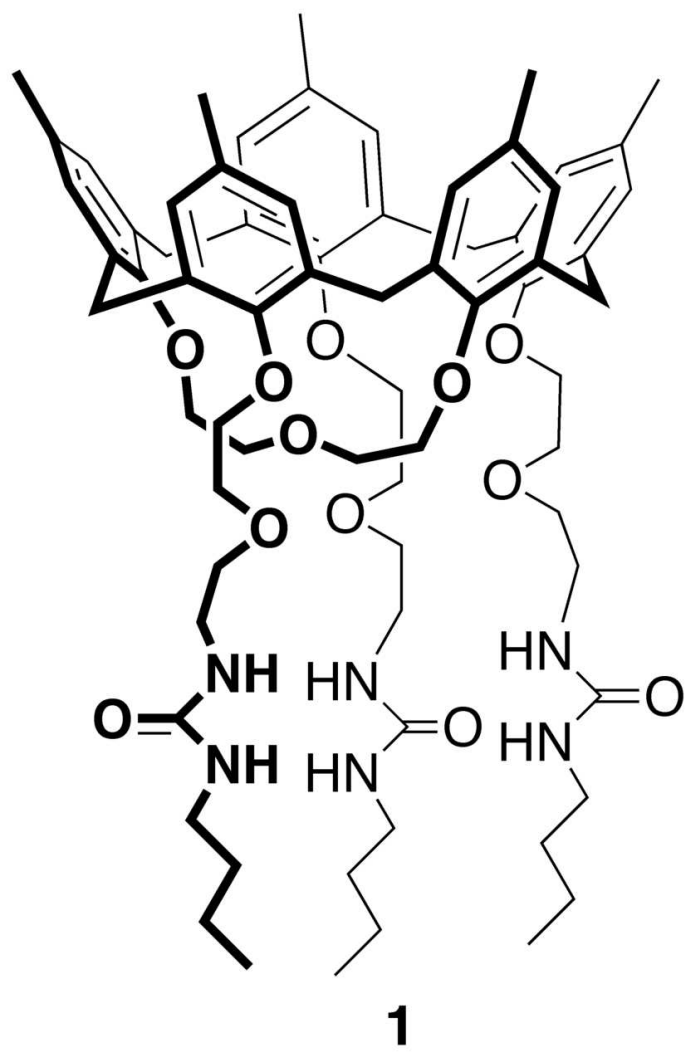

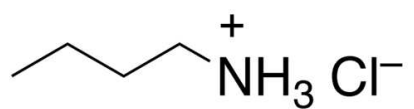

$2 \cdot \mathrm{HCl}$<smiles>[NH3+]CCc1ccccc1</smiles>

$3 \cdot \mathrm{HCl}$

$66 \times 53 \mathrm{~mm}(600 \times 600 \mathrm{DPI})$ 


\section{Supramolecular Chemistry}

\section{A DFT study on a calix[5]crown-based heteroditopic receptor}

Claudia Gargiulli ${ }^{\mathrm{a}}$, Giuseppe Gattuso ${ }^{\mathrm{a}}$, Anna Notti ${ }^{\mathrm{a}}$, Sebastiano Pappalardo ${ }^{b}$ and Melchiorre F. Parisi ${ }^{a}$

${ }^{a}$ Dipartimento di Chimica Organica e Biologica, Università di Messina, salita Sperone 31, 98166 Messina, Italy; ${ }^{b}$ Dipartimento di Scienze Chimiche, Università di Catania, Viale A. Doria 6, 95125 Catania, Italy

* Corresponding author. Email: gg@isengard.unime.it

\section{Supporting Information}


Table 1. DFT B3LYP/6-311++G(d,p)//B3LYP/6-31G(d) calculated energies (A.U. $)^{\mathrm{a}}$ for calix[5]crown 1 and its complexes.

\begin{tabular}{lc}
\hline & $\mathrm{B} 3 \mathrm{LYP} / 6-311++\mathrm{G}(\mathrm{d}, \mathrm{p})$ \\
\hline $\mathbf{1}$ & -3998.0997 \\
{$\left[\mathbf{1} \supset \mathrm{Cl}^{-}\right]$} & -4458.4886 \\
{$\left[\mathbf{2} \cdot \mathrm{H}^{+} \subset \mathbf{1}\right]$} & -4212.3830 \\
{$\left[\mathbf{2} \cdot \mathrm{H}^{+} \subset \mathbf{1} \supset \mathrm{Cl}^{-}\right]$} & -4672.8334 \\
$\left\{\left[\mathbf{1} \supset \mathrm{Cl}^{-}\right]-\mathrm{Cl}^{-}\right\}$ & -3998.0848 \\
$\left\{\left[\mathbf{2} \cdot \mathrm{H}^{+} \subset \mathbf{1}\right]-\mathbf{2} \cdot \mathrm{H}^{+}\right\}$ & -3998.0842 \\
$\left\{\left[\mathbf{2} \cdot \mathrm{H}^{+} \subset \mathbf{1} \supset \mathrm{Cl}^{-}\right]-\mathrm{Cl}^{-}\right\}$ & -4212.3540 \\
$\left\{\left[\mathbf{2} \cdot \mathrm{H}^{+} \subset \mathbf{1} \supset \mathrm{Cl}^{-}\right]-\mathbf{2} \cdot \mathrm{H}^{+}\right\}$ & -4458.4724 \\
$\left\{\left[\mathbf{2} \cdot \mathrm{H}^{+} \subset \mathbf{1} \supset \mathrm{Cl}^{-}\right]-\mathbf{2} \cdot \mathrm{HCl}\right\}$ & -3998.0568 \\
{$\left[\mathbf{3} \cdot \mathrm{H}^{+} \subset \mathbf{1}\right]$} & -4364.8388 \\
{$\left[\mathbf{3} \cdot \mathrm{H}^{+} \subset \mathbf{1} \supset \mathrm{Cl}^{-}\right]$} & -4825.2880 \\
$\left\{\left[\mathbf{3} \cdot \mathrm{H}^{+} \subset \mathbf{1}\right]-\mathbf{3} \cdot \mathrm{H}^{+}\right\}$ & -3998.0880 \\
$\left\{\left[\mathbf{3} \cdot \mathrm{H}^{+} \subset \mathbf{1} \supset \mathrm{Cl}^{-}\right]-\mathrm{Cl}^{-}\right\}$ & -4364.8146 \\
$\left\{\left[\mathbf{3} \cdot \mathrm{H}^{+} \subset \mathbf{1} \supset \mathrm{Cl}^{-}\right]-\mathbf{3} \cdot \mathrm{H}^{+}\right\}$ & -4458.4752 \\
$\left\{\left[\mathbf{3} \cdot \mathrm{H}^{+} \subset \mathbf{1} \supset \mathrm{Cl}^{-}\right]-\mathbf{3} \cdot \mathrm{HCl}^{+}\right\}$ & -3998.0670 \\
$\mathbf{2} \cdot \mathrm{H}^{+}$ & -214.2338 \\
$\left\{\left[\mathbf{2} \cdot \mathrm{H}^{+} \subset \mathbf{1}\right]-\mathbf{1}\right\}$ & -214.2332 \\
$\left\{\left[\mathbf{2} \cdot \mathrm{H}^{+} \subset \mathbf{1} \supset \mathrm{Cl}^{-}\right]-\mathbf{1}-\mathrm{Cl}^{-}\right\}$ & -214.2319 \\
$\mathbf{3} \cdot \mathrm{H}^{+}$ & -366.6907 \\
$\left\{\left[\mathbf{3} \cdot \mathrm{H}^{+} \subset \mathbf{1}\right]-\mathbf{1}\right\}$ & -366.6897 \\
$\left\{\left[\mathbf{3} \cdot \mathrm{H}^{+} \subset \mathbf{1} \supset \mathrm{Cl}^{-}\right]-\mathbf{1}-\mathrm{Cl}^{-}\right\}$ & -366.6897 \\
$\mathrm{Cl}$ & -460.3037 \\
\hline $\mathbf{1} 0^{-5} \mathrm{~A} \mathrm{U}-$ &
\end{tabular}

${ }^{\text {a }}$ Error limit: $2 \times 10^{-5}$ A.U.. 

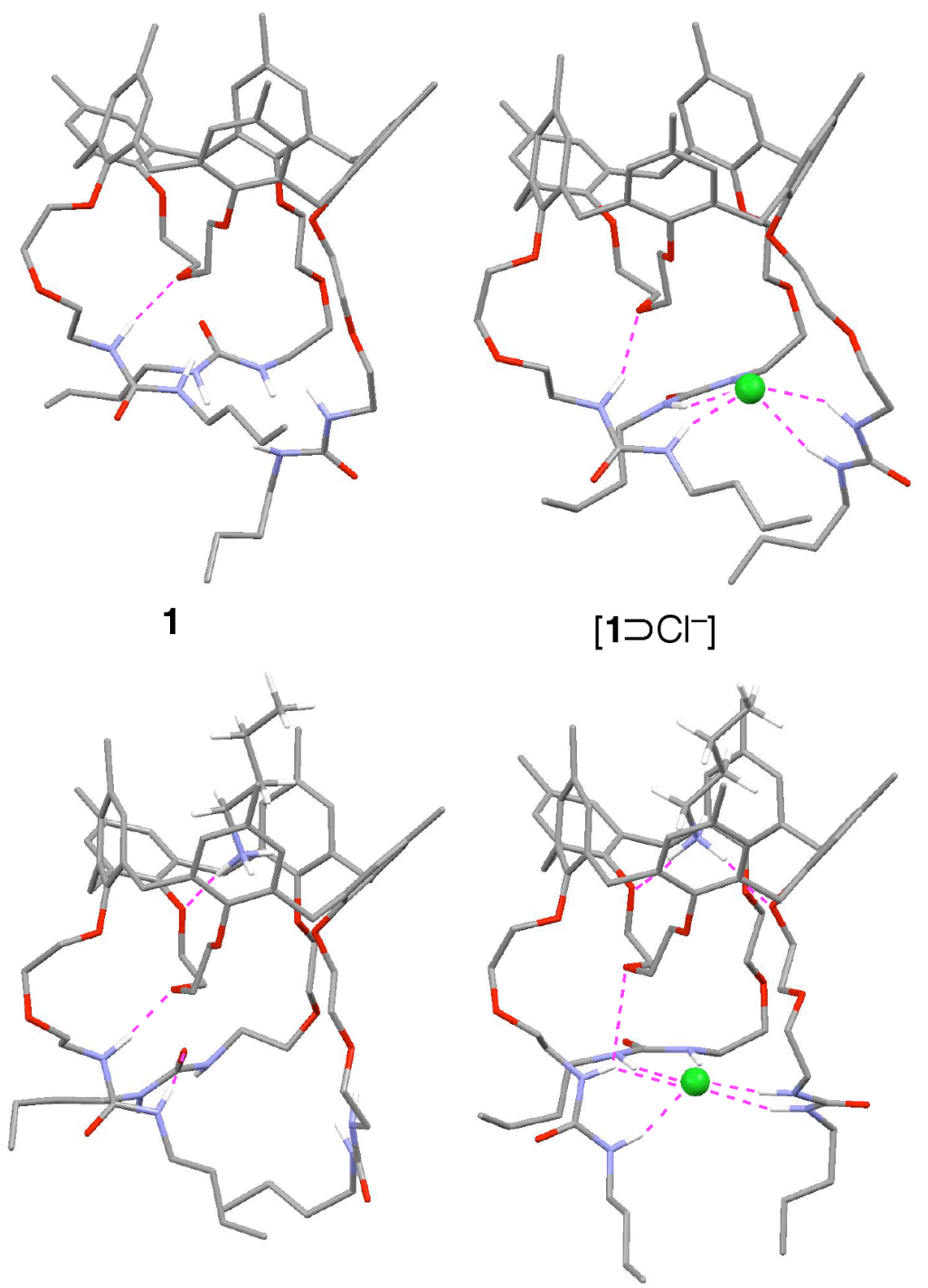

$\left[2 \cdot \mathrm{H}^{+} \subset 1\right]$

$\left[2 \cdot \mathrm{H}^{+} \subset 1 \supset \mathrm{Cl}^{-}\right]$

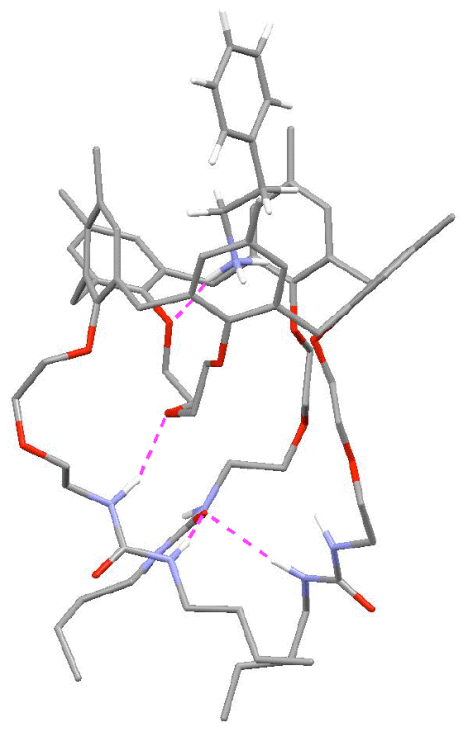

$\left[3 \cdot H^{+} \subset 1\right]$

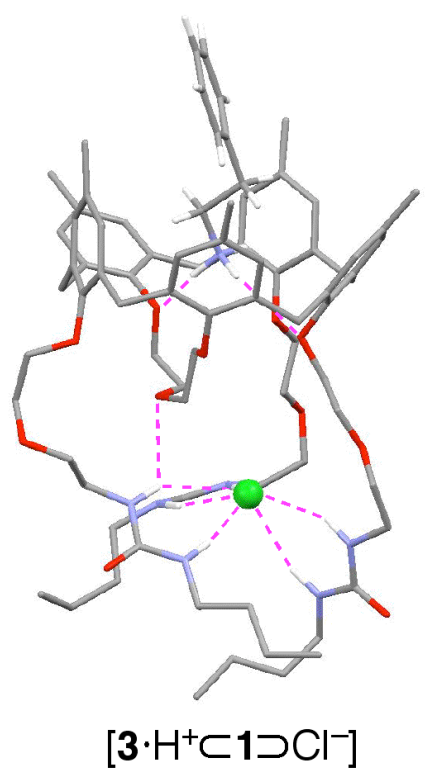

Figure 1. Optimised B3LYP/6-31-G(d) geometries for calix[5]crown $\mathbf{1}$ and its complexes with $\mathbf{2} \cdot \mathrm{H}^{+}$, $\mathbf{3} \cdot \mathrm{H}^{+}, \mathrm{Cl}^{-}, \mathbf{2} \cdot \mathrm{HCl}$ and $\mathbf{3} \cdot \mathrm{HCl}$. Hydrogen bonds represented as dashed lines. 


\section{Coordinates for $\mathbf{1}$}

\begin{tabular}{|c|c|c|c|}
\hline $\mathrm{O}$ & 0.587000 & 2.414000 & 2.481000 \\
\hline $\mathrm{C}$ & 1.972000 & 2.466000 & 2.583000 \\
\hline $\mathrm{C}$ & 2.686000 & 3.390000 & 1.802000 \\
\hline $\mathrm{C}$ & 4.083000 & 3.374000 & 1.867000 \\
\hline $\mathrm{C}$ & 4.775000 & 2.484000 & 2.692000 \\
\hline $\mathrm{O}$ & 0.898000 & 2.673000 & -1.141000 \\
\hline $\mathrm{C}$ & 4.032000 & 1.578000 & 3.454000 \\
\hline $\mathrm{C}$ & 1.896000 & 3.578000 & -1.482000 \\
\hline $\mathrm{C}$ & 2.636000 & 1.546000 & 3.414000 \\
\hline $\mathrm{C}$ & 2.431000 & 3.536000 & -2.780000 \\
\hline $\mathrm{C}$ & 3.389000 & 4.502000 & -3.122000 \\
\hline $\mathrm{C}$ & 3.847000 & 5.457000 & -2.216000 \\
\hline $\mathrm{C}$ & 3.346000 & 5.411000 & -0.910000 \\
\hline $\mathrm{C}$ & 2.389000 & 4.475000 & -0.512000 \\
\hline $\mathrm{C}$ & 4.850000 & 6.513000 & -2.624000 \\
\hline $\mathrm{C}$ & 1.962000 & 4.428000 & 0.948000 \\
\hline $\mathrm{C}$ & 6.284000 & 2.518000 & 2.781000 \\
\hline $\mathrm{C}$ & 2.021000 & 2.471000 & -3.794000 \\
\hline $\mathrm{C}$ & 1.857000 & 0.555000 & 4.275000 \\
\hline $\mathrm{O}$ & 1.601000 & -0.265000 & -3.026000 \\
\hline $\mathrm{C}$ & 2.852000 & 0.060000 & -3.513000 \\
\hline $\mathrm{C}$ & 3.842000 & -0.935000 & -3.676000 \\
\hline $\mathrm{C}$ & 5.007000 & -0.604000 & -4.374000 \\
\hline $\mathrm{C}$ & 5.230000 & 0.674000 & -4.895000 \\
\hline $\mathrm{C}$ & 4.249000 & 1.642000 & -4.680000 \\
\hline $\mathrm{C}$ & 3.062000 & 1.368000 & -3.989000 \\
\hline $\mathrm{C}$ & 6.475000 & 0.982000 & -5.695000 \\
\hline $\mathrm{C}$ & 3.692000 & -2.339000 & -3.113000 \\
\hline $\mathrm{O}$ & 2.334000 & -3.891000 & -1.081000 \\
\hline $\mathrm{C}$ & 3.471000 & -3.180000 & -0.713000 \\
\hline $\mathrm{C}$ & 3.809000 & -3.128000 & 0.651000 \\
\hline $\mathrm{C}$ & 4.990000 & -2.477000 & 1.013000 \\
\hline $\mathrm{C}$ & 5.803000 & -1.847000 & 0.066000 \\
\hline $\mathrm{C}$ & 5.376000 & -1.826000 & -1.263000 \\
\hline $\mathrm{C}$ & 4.204000 & -2.469000 & -1.677000 \\
\hline $\mathrm{C}$ & 7.067000 & -1.134000 & 0.487000 \\
\hline $\mathrm{O}$ & 1.567000 & -1.178000 & 1.977000 \\
\hline $\mathrm{C}$ & 2.253000 & -1.667000 & 3.077000 \\
\hline $\mathrm{C}$ & 2.384000 & -0.870000 & 4.228000 \\
\hline $\mathrm{C}$ & 3.067000 & -1.400000 & 5.328000 \\
\hline $\mathrm{C}$ & 3.638000 & -2.677000 & 5.306000 \\
\hline $\mathrm{C}$ & 3.542000 & -3.414000 & 4.121000 \\
\hline $\mathrm{C}$ & 2.865000 & -2.930000 & 2.996000 \\
\hline $\mathrm{C}$ & 4.328000 & -3.241000 & 6.526000 \\
\hline $\mathrm{C}$ & 2.866000 & -3.714000 & 1.699000 \\
\hline $\mathrm{H}$ & 4.643000 & 4.076000 & 1.253000 \\
\hline $\mathrm{H}$ & 4.551000 & 0.863000 & 4.088000 \\
\hline $\mathrm{H}$ & 3.779000 & 4.517000 & -4.137000 \\
\hline $\mathrm{H}$ & 3.712000 & 6.127000 & -0.175000 \\
\hline $\mathrm{H}$ & 2.152000 & 5.419000 & 1.380000 \\
\hline $\mathrm{H}$ & 0.892000 & 4.253000 & 1.041000 \\
\hline $\mathrm{H}$ & 1.853000 & 2.960000 & -4.762000 \\
\hline $\mathrm{H}$ & 1.082000 & 2.011000 & -3.490000 \\
\hline $\mathrm{H}$ & 1.892000 & 0.891000 & 5.319000 \\
\hline $\mathrm{H}$ & 0.807000 & 0.596000 & 3.980000 \\
\hline $\mathrm{H}$ & 5.756000 & -1.379000 & -4.531000 \\
\hline $\mathrm{H}$ & 4.406000 & 2.641000 & -5.078000 \\
\hline $\mathrm{H}$ & 4.240000 & -3.032000 & -3.765000 \\
\hline $\mathrm{H}$ & 2.644000 & -2.645000 & -3.143000 \\
\hline $\mathrm{H}$ & 5.266000 & -2.438000 & 2.063000 \\
\hline $\mathrm{H}$ & 5.954000 & -1.267000 & -1.994000 \\
\hline $\mathrm{H}$ & 3.156000 & -0.793000 & 6.227000 \\
\hline $\mathrm{H}$ & 4.011000 & -4.395000 & 4.065000 \\
\hline $\mathrm{H}$ & 3.140000 & -4.756000 & 1.917000 \\
\hline $\mathrm{H}$ & 1.864000 & -3.742000 & 1.263000 \\
\hline $\mathrm{C}$ & 2.604000 & -5.216000 & -1.575000 \\
\hline $\mathrm{C}$ & -0.156000 & 3.211000 & 3.412000 \\
\hline $\mathrm{C}$ & 0.140000 & -1.288000 & 2.070000 \\
\hline $\mathrm{C}$ & 1.399000 & -0.478000 & -1.618000 \\
\hline $\mathrm{C}$ & -0.404000 & 3.206000 & -0.893000 \\
\hline $\mathrm{C}$ & -1.316000 & 3.171000 & -2.119000 \\
\hline $\mathrm{H}$ & -0.350000 & 4.236000 & -0.530000 \\
\hline $\mathrm{H}$ & -0.847000 & 2.590000 & -0.106000 \\
\hline $\mathrm{H}$ & 0.022000 & 4.280000 & 3.239000 \\
\hline
\end{tabular}

$\begin{array}{llll}\text { C } & -1.621000 & 2.866000 & 3.181000\end{array}$

$\mathrm{H} \quad-1.784000 \quad 1.804000 \quad 3.397000$

$\begin{array}{llll}\mathrm{O} & -2.401000 & 3.691000 & 4.029000\end{array}$

$\mathrm{H} \quad-1.283000 \quad 2.187000 \quad-2.610000$

$\begin{array}{llll}\mathrm{O} & -2.616000 & 3.444000 & -1.618000\end{array}$

$\mathrm{H} \quad 1.902000 \quad-1.381000 \quad-1.274000$

C $\quad-0.108000 \quad-0.674000 \quad-1.480000$

$\begin{array}{llll}\mathrm{H} & 1.761000 & 0.379000 & -1.043000\end{array}$

$\begin{array}{llll}\mathrm{O} & -0.483000 & -1.325000 & -0.270000\end{array}$

$\begin{array}{llll}\mathrm{H} & -0.436000 & -1.337000 & -2.287000\end{array}$

$\mathrm{H} \quad-0.638000 \quad 0.281000 \quad-1.590000$

$\mathrm{H} \quad-0.223000 \quad-0.871000 \quad 3.016000$

$\mathrm{H} \quad-0.162000 \quad-2.345000 \quad 2.033000$

$\begin{array}{llll}\text { C } & -0.493000 & -0.525000 & 0.923000\end{array}$

$\begin{array}{llll}\mathrm{H} & 0.049000 & 0.413000 & 0.775000\end{array}$

$\mathrm{H} \quad-1.536000 \quad-0.296000 \quad 1.180000$

C $\quad 1.380000 \quad-6.113000 \quad-1.415000$

$\mathrm{H} \quad 3.435000 \quad-5.650000 \quad-1.001000$

$\mathrm{H} \quad 2.899000 \quad-5.173000 \quad-2.632000$

O $\quad 0.367000 \quad-5.939000 \quad-2.389000$

$\mathrm{H} \quad 0.992000 \quad-5.998000 \quad-0.392000$

$\mathrm{H} \quad 1.713000 \quad-7.151000 \quad-1.529000$

$\begin{array}{llll}\text { C } & -0.448000 & -4.778000 & -2.230000\end{array}$

$\mathrm{H} \quad-0.921000 \quad-4.610000 \quad-3.202000$

$\mathrm{H} \quad 0.169000 \quad-3.905000 \quad-1.988000$

C $\quad-1.542000 \quad-4.954000 \quad-1.164000$

$\mathrm{H} \quad-1.114000 \quad-5.339000 \quad-0.231000$

$\begin{array}{llll}H & -1.114000 & -5.339000 & -0.231000 \\ H & -2.297000 & -5.663000 & -1.506000\end{array}$

$\begin{array}{llll}\mathrm{H} & -1.012000 & 3.928000 & -2.859000\end{array}$

$\begin{array}{llll}\mathrm{H} & -1.875000 & 3.050000 & 2.124000\end{array}$

C $\quad-3.781000 \quad 3.736000 \quad 3.719000$

$\begin{array}{llll}\text { C } & -3.607000 & 3.682000 & -2.607000\end{array}$

$\mathrm{H} \quad-3.630000 \quad 2.856000 \quad-3.336000$

$\begin{array}{llll}\mathrm{H} & -3.395000 & 4.614000 & -3.154000\end{array}$

$\begin{array}{llll}\text { C } & -4.940000 & 3.791000 & -1.885000\end{array}$

$\mathrm{H} \quad-4.918000 \quad 4.648000 \quad-1.197000$

$\begin{array}{llll}\mathrm{N} & -5.206000 & 2.544000 & -1.174000\end{array}$

$\begin{array}{llll}\mathrm{H} & -5.745000 & 3.969000 & -2.603000\end{array}$

$\mathrm{H} \quad-4.184000 \quad 4.541000 \quad 4.344000$

$\begin{array}{llll}\mathrm{C} & -4.572000 & 2.451000 & 4.020000\end{array}$

H $\quad-4.377000 \quad 2.134000 \quad-0.757000$

$\begin{array}{llll}\text { C } & -6.384000 & 2.423000 & -0.464000\end{array}$

$\begin{array}{llll}\mathrm{O} & -7.280000 & 3.254000 & -0.498000\end{array}$

$\begin{array}{llll}\mathrm{N} & -6.450000 & 1.249000 & 0.313000\end{array}$

$\begin{array}{llll}\mathrm{H} & -5.992000 & 0.460000 & -0.135000\end{array}$

$\begin{array}{llll}\text { C } & -7.748000 & 0.917000 & 0.925000\end{array}$

$\begin{array}{llll}\text { C } & -8.791000 & 0.312000 & -0.023000\end{array}$

$\begin{array}{llll}\mathrm{H} & -8.130000 & 1.846000 & 1.352000\end{array}$

$\begin{array}{llll}\mathrm{H} & -7.547000 & 0.228000 & 1.756000\end{array}$

$\begin{array}{llll}\text { C } & -8.414000 & -1.060000 & -0.596000\end{array}$

$\begin{array}{llll}\mathrm{H} & -8.986000 & 1.024000 & -0.834000\end{array}$

$\begin{array}{llll}\mathrm{H} & -9.732000 & 0.218000 & 0.539000\end{array}$

$\begin{array}{llll}\mathrm{H} & -7.492000 & -0.980000 & -1.191000\end{array}$

$\begin{array}{llll}\text { C } & -9.515000 & -1.664000 & -1.473000\end{array}$

$\mathrm{H} \quad-8.187000 \quad-1.750000 \quad 0.230000$

$\mathrm{H} \quad-9.220000 \quad-2.644000 \quad-1.862000$

$\mathrm{H} \quad-9.738000 \quad-1.017000 \quad-2.331000$

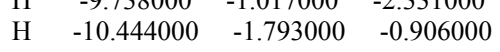

$\begin{array}{llll}\mathrm{N} & -2.203000 & -3.689000 & -0.873000\end{array}$

C $\quad-3.257000 \quad-3.265000 \quad-1.660000$

$\mathrm{H} \quad-1.603000 \quad-2.961000 \quad-0.489000$

$\begin{array}{llll}\mathrm{O} & -3.884000 & -4.000000 & -2.417000\end{array}$

$\begin{array}{llll}\mathrm{N} & -3.597000 & -1.926000 & -1.464000\end{array}$

$\begin{array}{llll}\text { C } & -4.463000 & -1.282000 & -2.445000\end{array}$

$\mathrm{H} \quad-2.864000 \quad-1.347000 \quad-1.068000$

$\mathrm{H} \quad-5.254000 \quad-1.998000 \quad-2.683000$

$\begin{array}{llll}\text { C } & -3.756000 & -0.837000 & -3.733000\end{array}$

$\mathrm{H} \quad-4.933000 \quad-0.409000 \quad-1.971000$

$\mathrm{H} \quad-3.301000 \quad-1.717000 \quad-4.206000$

$\mathrm{H} \quad-2.932000 \quad-0.154000 \quad-3.476000$

C $\quad-4.703000 \quad-0.148000 \quad-4.722000$

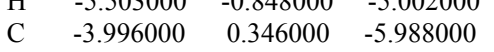

\begin{tabular}{|c|c|c|c|}
\hline $\mathrm{H}$ & -5.199000 & 0.697000 & -4.222000 \\
\hline $\mathrm{H}$ & -3.510000 & -0.481000 & -6.518000 \\
\hline $\mathrm{H}$ & -3.221000 & 1.085000 & -5.748000 \\
\hline $\mathrm{H}$ & -4.702000 & 0.817000 & -6.681000 \\
\hline $\mathrm{H}$ & 6.376000 & 0.646000 & -6.736000 \\
\hline $\mathrm{H}$ & 6.681000 & 2.058000 & -5.717000 \\
\hline $\mathrm{H}$ & 7.355000 & 0.480000 & -5.277000 \\
\hline $\mathrm{H}$ & 7.612000 & -1.699000 & 1.252000 \\
\hline $\mathrm{H}$ & 7.742000 & -0.978000 & -0.361000 \\
\hline $\mathrm{H}$ & 6.843000 & -0.146000 & 0.911000 \\
\hline $\mathrm{H}$ & 5.306000 & 6.277000 & -3.591000 \\
\hline $\mathrm{H}$ & 5.655000 & 6.606000 & -1.886000 \\
\hline $\mathrm{H}$ & 4.379000 & 7.501000 & -2.713000 \\
\hline $\mathrm{H}$ & 6.622000 & 3.143000 & 3.619000 \\
\hline $\mathrm{H}$ & 6.730000 & 2.929000 & 1.869000 \\
\hline $\mathrm{H}$ & 6.698000 & 1.517000 & 2.940000 \\
\hline $\mathrm{H}$ & 5.081000 & -3.988000 & 6.252000 \\
\hline $\mathrm{H}$ & 3.611000 & -3.734000 & 7.197000 \\
\hline $\mathrm{H}$ & 4.826000 & -2.457000 & 7.106000 \\
\hline $\mathrm{H}$ & -3.928000 & 4.023000 & 2.663000 \\
\hline $\mathrm{H}$ & -5.622000 & 2.733000 & 4.184000 \\
\hline $\mathrm{H}$ & -4.193000 & 2.007000 & 4.944000 \\
\hline $\mathrm{N}$ & -4.524000 & 1.424000 & 2.988000 \\
\hline $\mathrm{C}$ & -3.901000 & 0.215000 & 3.194000 \\
\hline $\mathrm{H}$ & -5.134000 & 1.527000 & 2.181000 \\
\hline $\mathrm{O}$ & -3.171000 & -0.012000 & 4.160000 \\
\hline $\mathrm{N}$ & -4.101000 & -0.717000 & 2.181000 \\
\hline $\mathrm{C}$ & -3.776000 & -2.125000 & 2.425000 \\
\hline $\mathrm{H}$ & -4.914000 & -0.566000 & 1.596000 \\
\hline $\mathrm{C}$ & -4.844000 & -2.870000 & 3.239000 \\
\hline $\mathrm{H}$ & -2.828000 & -2.140000 & 2.968000 \\
\hline $\mathrm{H}$ & -3.619000 & -2.597000 & 1.451000 \\
\hline $\mathrm{H}$ & -4.983000 & -2.333000 & 4.185000 \\
\hline $\mathrm{C}$ & -4.486000 & -4.337000 & 3.532000 \\
\hline $\mathrm{H}$ & -5.803000 & -2.831000 & 2.701000 \\
\hline $\mathrm{H}$ & -5.212000 & -4.733000 & 4.254000 \\
\hline $\mathrm{H}$ & -3.509000 & -4.374000 & 4.036000 \\
\hline $\mathrm{C}$ & -4.465000 & -5.243000 & 2.294000 \\
\hline $\mathrm{H}$ & -5.435000 & -5.227000 & 1.780000 \\
\hline $\mathrm{H}$ & -4.256000 & -6.282000 & 2.573000 \\
\hline $\mathrm{H}$ & -3.705000 & -4.937000 & 1.567000 \\
\hline
\end{tabular}




\section{Coordinates for $\left[1 \supset \mathrm{Cl}^{-}\right]$}

\begin{tabular}{|c|c|c|c|c|c|c|c|c|c|c|c|}
\hline $\mathrm{O}$ & 1.017000 & 0.503000 & 3.302000 & $\mathrm{H}$ & 0.299000 & 1.448000 & 5.021000 & $\mathrm{H}$ & -6.757000 & 1.671000 & -4.318000 \\
\hline $\mathrm{C}$ & 2.398000 & 0.585000 & 3.375000 & $\mathrm{H}$ & 0.750000 & -0.258000 & 5.233000 & $\mathrm{C}$ & -6.377000 & 3.564000 & -3.335000 \\
\hline $\mathrm{C}$ & 3.019000 & 1.837000 & 3.234000 & $\mathrm{C}$ & -1.125000 & 0.032000 & 4.192000 & $\mathrm{H}$ & -7.210000 & 1.707000 & -2.620000 \\
\hline $\mathrm{C}$ & 4.417000 & 1.887000 & 3.227000 & $\mathrm{H}$ & -1.099000 & -0.925000 & 3.658000 & $\mathrm{H}$ & -5.615000 & 3.928000 & -4.037000 \\
\hline $\mathrm{C}$ & 5.199000 & 0.738000 & 3.358000 & $\mathrm{O}$ & -1.845000 & -0.071000 & 5.410000 & $\mathrm{H}$ & -6.126000 & 3.943000 & -2.338000 \\
\hline $\mathrm{O}$ & 1.022000 & 2.813000 & 0.474000 & $\mathrm{H}$ & -1.212000 & 3.460000 & -1.045000 & $\mathrm{H}$ & -7.338000 & 4.003000 & -3.629000 \\
\hline $\mathrm{C}$ & 4.548000 & -0.493000 & 3.489000 & $\mathrm{O}$ & -2.245000 & 4.431000 & 0.474000 & $\mathrm{H}$ & 6.528000 & 4.988000 & -4.102000 \\
\hline $\mathrm{C}$ & 2.020000 & 3.757000 & 0.634000 & $\mathrm{H}$ & 2.100000 & -0.469000 & -1.885000 & $\mathrm{H}$ & 7.158000 & 3.476000 & -4.776000 \\
\hline $\mathrm{C}$ & 3.156000 & -0.595000 & 3.498000 & $\mathrm{C}$ & 0.094000 & 0.151000 & -1.475000 & $\mathrm{H}$ & 6.029000 & 4.459000 & -5.712000 \\
\hline $\mathrm{C}$ & 2.515000 & 4.442000 & -0.489000 & $\mathrm{H}$ & 2.023000 & 0.882000 & -0.725000 & $\mathrm{H}$ & 8.373000 & -1.675000 & -1.497000 \\
\hline $\mathrm{C}$ & 3.476000 & 5.442000 & -0.279000 & $\mathrm{O}$ & -0.121000 & -1.159000 & -0.962000 & $\mathrm{H}$ & 7.780000 & -0.052000 & -1.128000 \\
\hline $\mathrm{C}$ & 3.968000 & 5.753000 & 0.987000 & $\mathrm{H}$ & -0.356000 & 0.162000 & -2.473000 & $\mathrm{H}$ & 7.659000 & -1.340000 & 0.083000 \\
\hline $\mathrm{C}$ & 3.511000 & 4.994000 & 2.070000 & $\mathrm{H}$ & -0.421000 & 0.897000 & -0.863000 & $\mathrm{H}$ & 5.479000 & 7.126000 & 0.261000 \\
\hline $\mathrm{C}$ & 2.562000 & 3.981000 & 1.918000 & $\mathrm{H}$ & 0.411000 & -2.841000 & 1.860000 & $\mathrm{H}$ & 5.707000 & 6.625000 & 1.945000 \\
\hline $\mathrm{C}$ & 4.954000 & 6.881000 & 1.190000 & $\mathrm{H}$ & 0.489000 & -3.348000 & 0.163000 & $\mathrm{H}$ & 4.453000 & 7.797000 & 1.533000 \\
\hline $\mathrm{C}$ & 2.188000 & 3.113000 & 3.109000 & $\mathrm{C}$ & -0.118000 & -1.303000 & 0.463000 & $\mathrm{H}$ & 7.106000 & 0.821000 & 4.393000 \\
\hline $\mathrm{C}$ & 6.708000 & 0.820000 & 3.369000 & $\mathrm{H}$ & 0.290000 & -0.414000 & 0.953000 & $\mathrm{H}$ & 7.062000 & 1.734000 & 2.880000 \\
\hline $\mathrm{C}$ & 2.039000 & 4.122000 & -1.904000 & $\mathrm{H}$ & -1.149000 & -1.439000 & 0.808000 & $\mathrm{H}$ & 7.159000 & -0.034000 & 2.849000 \\
\hline $\mathrm{C}$ & 2.475000 & -1.951000 & 3.651000 & $\mathrm{C}$ & 1.279000 & -4.358000 & -4.302000 & $\mathrm{H}$ & 5.370000 & -7.030000 & 2.881000 \\
\hline $\mathrm{O}$ & 1.664000 & 1.408000 & -2.715000 & $\mathrm{H}$ & 3.399000 & -4.301000 & -3.954000 & $\mathrm{H}$ & 5.157000 & -6.189000 & 4.425000 \\
\hline $\mathrm{C}$ & 2.864000 & 1.990000 & -3.052000 & $\mathrm{H}$ & 2.822000 & -3.082000 & -5.120000 & $\mathrm{H}$ & 6.491000 & -5.751000 & 3.353000 \\
\hline $\mathrm{C}$ & 3.815000 & 1.290000 & -3.828000 & $\mathrm{O}$ & 0.243000 & -3.674000 & -4.975000 & $\mathrm{H}$ & -3.545000 & 0.853000 & 4.660000 \\
\hline $\mathrm{C}$ & 4.909000 & 1.996000 & -4.337000 & $\mathrm{H}$ & 0.970000 & -4.700000 & -3.304000 & $\mathrm{H}$ & -5.012000 & -1.197000 & 4.996000 \\
\hline $\mathrm{C}$ & 5.104000 & 3.357000 & -4.087000 & $\mathrm{H}$ & 1.483000 & -5.251000 & -4.908000 & $\mathrm{H}$ & -3.563000 & -2.153000 & 5.312000 \\
\hline $\mathrm{C}$ & 4.167000 & 4.009000 & -3.284000 & $\mathrm{C}$ & -0.505000 & -2.732000 & -4.196000 & $\mathrm{~N}$ & -3.697000 & -1.497000 & 3.358000 \\
\hline $\mathrm{C}$ & 3.050000 & 3.354000 & -2.751000 & $\mathrm{H}$ & -1.045000 & -2.120000 & -4.924000 & $\mathrm{C}$ & -3.763000 & -2.758000 & 2.802000 \\
\hline $\mathrm{C}$ & 6.265000 & 4.107000 & -4.698000 & $\mathrm{H}$ & 0.165000 & -2.085000 & -3.619000 & $\mathrm{H}$ & -3.710000 & -0.697000 & 2.727000 \\
\hline $\mathrm{C}$ & 3.684000 & -0.195000 & -4.119000 & $\mathrm{C}$ & -1.530000 & -3.394000 & -3.262000 & $\mathrm{O}$ & -3.932000 & -3.780000 & 3.472000 \\
\hline $\mathrm{O}$ & 2.506000 & -2.628000 & -3.108000 & $\mathrm{H}$ & -1.027000 & -4.044000 & -2.536000 & $\mathrm{~N}$ & -3.563000 & -2.766000 & 1.437000 \\
\hline $\mathrm{C}$ & 3.730000 & -2.236000 & -2.584000 & $\mathrm{H}$ & -2.221000 & -4.002000 & -3.848000 & $\mathrm{C}$ & -3.833000 & -3.955000 & 0.643000 \\
\hline $\mathrm{C}$ & 4.260000 & -2.956000 & -1.498000 & $\mathrm{H}$ & -0.437000 & 4.940000 & -0.417000 & $\mathrm{H}$ & -3.588000 & -1.865000 & 0.963000 \\
\hline $\mathrm{C}$ & 5.508000 & -2.578000 & -0.999000 & $\mathrm{H}$ & -1.585000 & 0.768000 & 3.516000 & $\mathrm{C}$ & -5.301000 & -4.077000 & 0.202000 \\
\hline $\mathrm{C}$ & 6.210000 & -1.489000 & -1.522000 & $\mathrm{C}$ & -3.260000 & -0.008000 & 5.288000 & $\mathrm{H}$ & -3.554000 & -4.821000 & 1.252000 \\
\hline $\mathrm{C}$ & 5.607000 & -0.737000 & -2.533000 & $\mathrm{C}$ & -3.181000 & 5.060000 & -0.386000 & $\mathrm{H}$ & -3.177000 & -3.923000 & -0.233000 \\
\hline $\mathrm{C}$ & 4.363000 & -1.082000 & -3.074000 & $\mathrm{H}$ & -3.247000 & 4.516000 & -1.341000 & $\mathrm{H}$ & -5.926000 & -4.053000 & 1.103000 \\
\hline $\mathrm{C}$ & 7.574000 & -1.118000 & -0.987000 & $\mathrm{H}$ & -2.875000 & 6.098000 & -0.601000 & $\mathrm{C}$ & -5.617000 & -5.348000 & -0.603000 \\
\hline $\mathrm{O}$ & 2.102000 & -2.193000 & 0.819000 & $\mathrm{C}$ & -4.527000 & 5.037000 & 0.318000 & $\mathrm{H}$ & -5.566000 & -3.192000 & -0.395000 \\
\hline $\mathrm{C}$ & 2.912000 & -3.124000 & 1.437000 & $\mathrm{H}$ & -4.442000 & 5.574000 & 1.276000 & $\mathrm{H}$ & -6.700000 & -5.381000 & -0.786000 \\
\hline $\mathrm{C}$ & 3.102000 & -3.061000 & 2.829000 & $\mathrm{~N}$ & -4.948000 & 3.660000 & 0.500000 & $\mathrm{H}$ & -5.389000 & -6.230000 & 0.014000 \\
\hline $\mathrm{C}$ & 3.912000 & -4.028000 & 3.435000 & $\mathrm{H}$ & -5.272000 & 5.569000 & -0.282000 & $\mathrm{C}$ & -4.882000 & -5.461000 & -1.946000 \\
\hline $\mathrm{C}$ & 4.550000 & -5.031000 & 2.698000 & $\mathrm{H}$ & -3.628000 & 0.185000 & 6.303000 & $\mathrm{H}$ & -5.040000 & -4.576000 & -2.574000 \\
\hline $\mathrm{C}$ & 4.372000 & -5.038000 & 1.311000 & $\mathrm{C}$ & -3.934000 & -1.280000 & 4.768000 & $\mathrm{H}$ & -5.228000 & -6.337000 & -2.508000 \\
\hline $\mathrm{C}$ & 3.570000 & -4.094000 & 0.661000 & $\mathrm{H}$ & -4.202000 & 2.983000 & 0.652000 & $\mathrm{H}$ & -3.800000 & -5.569000 & -1.808000 \\
\hline $\mathrm{C}$ & 5.435000 & -6.053000 & 3.374000 & $\mathrm{C}$ & -6.166000 & 3.402000 & 1.103000 & $\mathrm{Cl}$ & -3.363000 & 0.558000 & 0.691000 \\
\hline $\mathrm{C}$ & 3.454000 & -4.079000 & -0.851000 & $\mathrm{O}$ & -7.038000 & 4.268000 & 1.227000 & & & & \\
\hline $\mathrm{H}$ & 4.907000 & 2.848000 & 3.093000 & $\mathrm{~N}$ & -6.305000 & 2.106000 & 1.540000 & & & & \\
\hline $\mathrm{H}$ & 5.139000 & -1.403000 & 3.563000 & $\mathrm{H}$ & -5.556000 & 1.448000 & 1.334000 & & & & \\
\hline $\mathrm{H}$ & 3.834000 & 6.009000 & -1.135000 & $\mathrm{C}$ & -7.563000 & 1.618000 & 2.068000 & & & & \\
\hline $\mathrm{H}$ & 3.904000 & 5.196000 & 3.066000 & $\mathrm{C}$ & -8.394000 & 0.793000 & 1.071000 & & & & \\
\hline $\mathrm{H}$ & 2.323000 & 3.709000 & 4.021000 & $\mathrm{H}$ & -8.130000 & 2.499000 & 2.380000 & & & & \\
\hline $\mathrm{H}$ & 1.138000 & 2.825000 & 3.073000 & $\mathrm{H}$ & -7.362000 & 1.012000 & 2.964000 & & & & \\
\hline $\mathrm{H}$ & 1.809000 & 5.066000 & -2.414000 & $\mathrm{C}$ & -7.728000 & -0.520000 & 0.643000 & & & & \\
\hline $\mathrm{H}$ & 1.120000 & 3.540000 & -1.860000 & $\mathrm{H}$ & -8.596000 & 1.415000 & 0.187000 & & & & \\
\hline $\mathrm{H}$ & 2.503000 & -2.249000 & 4.708000 & $\mathrm{H}$ & -9.370000 & 0.574000 & 1.531000 & & & & \\
\hline $\mathrm{H}$ & 1.422000 & -1.831000 & 3.394000 & $\mathrm{H}$ & -6.741000 & -0.308000 & 0.213000 & & & & \\
\hline $\mathrm{H}$ & 5.622000 & 1.465000 & -4.967000 & $\mathrm{C}$ & -8.562000 & -1.317000 & -0.366000 & & & & \\
\hline $\mathrm{H}$ & 4.298000 & 5.070000 & -3.085000 & $\mathrm{H}$ & -7.543000 & -1.139000 & 1.533000 & & & & \\
\hline $\mathrm{H}$ & 4.125000 & -0.394000 & -5.106000 & $\mathrm{H}$ & -8.063000 & -2.252000 & -0.644000 & & & & \\
\hline $\mathrm{H}$ & 2.629000 & -0.471000 & -4.186000 & $\mathrm{H}$ & -8.727000 & -0.741000 & -1.285000 & & & & \\
\hline $\mathrm{H}$ & 5.924000 & -3.127000 & -0.158000 & $\mathrm{H}$ & -9.548000 & -1.572000 & 0.045000 & & & & \\
\hline $\mathrm{H}$ & 6.101000 & 0.163000 & -2.888000 & $\mathrm{~N}$ & -2.305000 & -2.406000 & -2.531000 & & & & \\
\hline $\mathrm{H}$ & 4.046000 & -3.994000 & 4.515000 & $\mathrm{C}$ & -3.444000 & -1.866000 & -3.114000 & & & & \\
\hline $\mathrm{H}$ & 4.868000 & -5.802000 & 0.713000 & $\mathrm{H}$ & -1.769000 & -1.839000 & -1.876000 & & & & \\
\hline $\mathrm{H}$ & 3.784000 & -5.053000 & -1.239000 & $\mathrm{O}$ & -3.966000 & -2.366000 & -4.116000 & & & & \\
\hline $\mathrm{H}$ & 2.412000 & -3.956000 & -1.155000 & $\mathrm{~N}$ & -3.933000 & -0.752000 & -2.480000 & & & & \\
\hline $\mathrm{C}$ & 2.590000 & -3.589000 & -4.172000 & $\mathrm{C}$ & -5.191000 & -0.146000 & -2.883000 & & & & \\
\hline $\mathrm{C}$ & 0.295000 & 0.461000 & 4.539000 & $\mathrm{H}$ & -3.575000 & -0.483000 & -1.564000 & & & & \\
\hline $\mathrm{C}$ & 0.697000 & -2.518000 & 0.852000 & $\mathrm{H}$ & -5.445000 & -0.570000 & -3.858000 & & & & \\
\hline $\mathrm{C}$ & 1.585000 & 0.458000 & -1.632000 & $\mathrm{C}$ & -5.102000 & 1.382000 & -2.952000 & & & & \\
\hline $\mathrm{C}$ & -0.292000 & 3.214000 & 0.906000 & $\mathrm{H}$ & -5.995000 & -0.432000 & -2.185000 & & & & \\
\hline $\mathrm{C}$ & -1.029000 & 4.053000 & -0.136000 & $\mathrm{H}$ & -4.328000 & 1.660000 & -3.680000 & & & & \\
\hline $\mathrm{H}$ & -0.233000 & 3.791000 & 1.836000 & $\mathrm{H}$ & -4.773000 & 1.768000 & -1.978000 & & & & \\
\hline $\mathrm{H}$ & -0.852000 & 2.295000 & 1.096000 & $\mathrm{C}$ & -6.437000 & 2.033000 & -3.330000 & & & & \\
\hline
\end{tabular}


Coordinates for $\left[\mathbf{2} \cdot \mathrm{H}^{+} \subset \mathbf{1}\right]$

\begin{tabular}{|c|c|c|c|}
\hline $\mathrm{O}$ & 0.355000 & 2.010000 & 2.575000 \\
\hline $\mathrm{C}$ & 1.646000 & 1.894000 & 3.059000 \\
\hline $\mathrm{C}$ & 2.582000 & 2.907000 & 2.763000 \\
\hline $\mathrm{C}$ & 3.894000 & 2.756000 & 3.235000 \\
\hline $\mathrm{C}$ & 4.296000 & 1.640000 & 3.977000 \\
\hline $\mathrm{O}$ & 1.458000 & 2.786000 & -0.494000 \\
\hline $\mathrm{C}$ & 3.353000 & 0.630000 & 4.206000 \\
\hline $\mathrm{C}$ & 2.569000 & 3.625000 & -0.534000 \\
\hline $\mathrm{C}$ & 2.031000 & 0.729000 & 3.760000 \\
\hline $\mathrm{C}$ & 3.311000 & 3.732000 & -1.724000 \\
\hline $\mathrm{C}$ & 4.379000 & 4.644000 & -1.735000 \\
\hline $\mathrm{C}$ & 4.751000 & 5.385000 & -0.613000 \\
\hline $\mathrm{C}$ & 4.030000 & 5.190000 & 0.571000 \\
\hline $\mathrm{C}$ & 2.939000 & 4.320000 & 0.640000 \\
\hline $\mathrm{C}$ & 5.879000 & 6.389000 & -0.674000 \\
\hline $\mathrm{C}$ & 2.196000 & 4.149000 & 1.963000 \\
\hline $\mathrm{C}$ & 5.696000 & 1.536000 & 4.537000 \\
\hline $\mathrm{C}$ & 2.992000 & 2.915000 & -2.976000 \\
\hline $\mathrm{C}$ & 1.039000 & -0.392000 & 4.046000 \\
\hline $\mathrm{O}$ & 2.045000 & 0.185000 & -3.410000 \\
\hline $\mathrm{C}$ & 3.416000 & 0.404000 & -3.383000 \\
\hline $\mathrm{C}$ & 4.290000 & -0.682000 & -3.629000 \\
\hline $\mathrm{C}$ & 5.647000 & -0.409000 & -3.817000 \\
\hline $\mathrm{C}$ & 6.172000 & 0.884000 & -3.714000 \\
\hline $\mathrm{C}$ & 5.293000 & 1.919000 & -3.396000 \\
\hline $\mathrm{C}$ & 3.914000 & 1.714000 & -3.227000 \\
\hline $\mathrm{C}$ & 7.637000 & 1.146000 & -3.969000 \\
\hline $\mathrm{C}$ & 3.796000 & -2.124000 & -3.696000 \\
\hline $\mathrm{O}$ & 1.614000 & -3.475000 & -2.309000 \\
\hline $\mathrm{C}$ & 2.836000 & -3.363000 & -1.670000 \\
\hline $\mathrm{C}$ & 2.933000 & -3.740000 & -0.313000 \\
\hline $\mathrm{C}$ & 4.207000 & -3.823000 & 0.256000 \\
\hline $\mathrm{C}$ & 5.366000 & -3.518000 & -0.470000 \\
\hline $\mathrm{C}$ & 5.211000 & -2.981000 & -1.750000 \\
\hline $\mathrm{C}$ & 3.953000 & -2.854000 & -2.358000 \\
\hline $\mathrm{C}$ & 6.736000 & -3.753000 & 0.123000 \\
\hline $\mathrm{O}$ & 0.983000 & -1.340000 & 1.312000 \\
\hline $\mathrm{C}$ & 1.394000 & -2.239000 & 2.304000 \\
\hline $\mathrm{C}$ & 1.467000 & -1.796000 & 3.637000 \\
\hline $\mathrm{C}$ & 1.899000 & -2.707000 & 4.608000 \\
\hline $\mathrm{C}$ & 2.246000 & -4.025000 & 4.289000 \\
\hline $\mathrm{C}$ & 2.149000 & -4.424000 & 2.952000 \\
\hline $\mathrm{C}$ & 1.735000 & -3.553000 & 1.937000 \\
\hline $\mathrm{C}$ & 2.716000 & -4.987000 & 5.355000 \\
\hline $\mathrm{C}$ & 1.671000 & -4.034000 & 0.495000 \\
\hline $\mathrm{H}$ & 4.617000 & 3.540000 & 3.023000 \\
\hline $\mathrm{H}$ & 3.653000 & -0.264000 & 4.749000 \\
\hline $\mathrm{H}$ & 4.919000 & 4.803000 & -2.665000 \\
\hline $\mathrm{H}$ & 4.308000 & 5.753000 & 1.459000 \\
\hline $\mathrm{H}$ & 2.408000 & 5.030000 & 2.579000 \\
\hline $\mathrm{H}$ & 1.119000 & 4.132000 & 1.806000 \\
\hline $\mathrm{H}$ & 3.071000 & 3.583000 & -3.844000 \\
\hline $\mathrm{H}$ & 1.961000 & 2.564000 & -2.952000 \\
\hline $\mathrm{H}$ & 0.840000 & -0.408000 & 5.125000 \\
\hline $\mathrm{H}$ & 0.095000 & -0.142000 & 3.564000 \\
\hline $\mathrm{H}$ & 6.314000 & -1.227000 & -4.078000 \\
\hline $\mathrm{H}$ & 5.689000 & 2.925000 & -3.309000 \\
\hline $\mathrm{H}$ & 4.360000 & -2.650000 & -4.476000 \\
\hline $\mathrm{H}$ & 2.748000 & -2.130000 & -3.996000 \\
\hline $\mathrm{H}$ & 4.300000 & -4.146000 & 1.290000 \\
\hline $\mathrm{H}$ & 6.093000 & -2.651000 & -2.292000 \\
\hline $\mathrm{H}$ & 1.943000 & -2.385000 & 5.647000 \\
\hline $\mathrm{H}$ & 2.396000 & -5.450000 & 2.687000 \\
\hline $\mathrm{H}$ & 1.483000 & -5.116000 & 0.503000 \\
\hline $\mathrm{H}$ & 0.829000 & -3.576000 & -0.027000 \\
\hline $\mathrm{C}$ & 1.567000 & -4.508000 & -3.333000 \\
\hline $\mathrm{C}$ & -0.642000 & 2.482000 & 3.502000 \\
\hline $\mathrm{C}$ & -0.459000 & -1.270000 & 1.139000 \\
\hline $\mathrm{C}$ & 1.448000 & -0.160000 & -2.161000 \\
\hline $\mathrm{C}$ & 0.177000 & 3.453000 & -0.484000 \\
\hline $\mathrm{C}$ & -0.478000 & 3.533000 & -1.859000 \\
\hline $\mathrm{H}$ & 0.286000 & 4.467000 & -0.091000 \\
\hline $\mathrm{H}$ & -0.462000 & 2.883000 & 0.192000 \\
\hline $\mathrm{H}$ & -0.389000 & 3.489000 & 3.855000 \\
\hline
\end{tabular}

$\begin{array}{lrrr}\mathrm{H} & -0.707000 & 1.819000 & 4.373000 \\ \mathrm{C} & -1.965000 & 2.460000 & 2.743000 \\ \mathrm{H} & -2.053000 & 1.492000 & 2.233000 \\ \mathrm{O} & -3.031000 & 2.660000 & 3.654000 \\ \mathrm{H} & -0.636000 & 2.524000 & -2.272000 \\ \mathrm{O} & -1.708000 & 4.189000 & -1.637000 \\ \mathrm{H} & 1.960000 & -1.014000 & -1.708000 \\ \mathrm{C} & -0.007000 & -0.560000 & -2.388000\end{array}$

$\begin{array}{llll}\mathrm{H} & 1.498000 & 0.706000 & -1.491000\end{array}$

$\begin{array}{llll}\mathrm{O} & -0.509000 & -1.238000 & -1.242000\end{array}$

$\mathrm{H} \quad-0.056000 \quad-1.276000 \quad-3.210000$

$\begin{array}{llll}\mathrm{H} & -0.632000 & 0.305000 & -2.648000\end{array}$

$\begin{array}{llll}\mathrm{H} & -0.922000 & -0.819000 & 2.020000\end{array}$

$\begin{array}{llll}\mathrm{H} & -0.859000 & -2.282000 & 1.026000\end{array}$

$\begin{array}{llll}\text { C } & -0.781000 & -0.446000 & -0.087000\end{array}$

$\begin{array}{llll}\mathrm{H} & -0.191000 & 0.476000 & -0.075000\end{array}$

$\mathrm{H} \quad-1.845000 \quad-0.176000-0.055000$

$\begin{array}{llll}\text { C } & 0.469000 & -5.549000 & -3.079000\end{array}$

$\mathrm{H} \quad 2.542000 \quad-5.005000 \quad-3.367000$

$\mathrm{H} \quad 1.391000 \quad-4.030000 \quad-4.304000$

$\begin{array}{llll}\mathrm{O} & -0.753000 & -5.297000 & -3.744000\end{array}$

$\mathrm{H} \quad 0.340000 \quad-5.673000 \quad-1.993000$

$\mathrm{H} \quad 0.808000 \quad-6.509000 \quad-3.483000$

C $\quad-1.489000 \quad-4.166000 \quad-3.291000$

$\mathrm{H} \quad-2.191000 \quad-3.921000 \quad-4.092000$

$\mathrm{H} \quad-0.822000 \quad-3.306000 \quad-3.142000$

C $\quad-2.274000 \quad-4.431000-1.998000$

$\mathrm{H} \quad-1.617000 \quad-4.862000 \quad-1.233000$

$\begin{array}{llll}\mathrm{H} & -3.079000 & -5.141000 & -2.194000\end{array}$

$\begin{array}{llll}\mathrm{H} & 0.149000 & 4.093000 & -2.570000\end{array}$

$\begin{array}{lrrr}\mathrm{H} & -1.976000 & 3.244000 & 1.970000\end{array}$

$\begin{array}{llll}\text { C } & -4.168000 & 1.846000 & 3.381000\end{array}$

C $\quad-2.631000 \quad 4.104000 \quad-2.724000$

$\mathrm{H} \quad-2.855000 \quad 3.048000 \quad-2.939000$

$\mathrm{H} \quad-2.199000 \quad 4.561000 \quad-3.629000$

$\mathrm{H} \quad-3.664000 \quad 5.916000 \quad-2.200000$

$\mathrm{N} \quad-4.441000 \quad 4.277000 \quad-1.093000$

$\begin{array}{llll}\mathrm{H} & -4.648000 & 4.756000 & -3.098000\end{array}$

$\begin{array}{llll}\mathrm{H} & -5.027000 & 2.344000 & 3.840000\end{array}$

$\begin{array}{llll}\text { C } & -3.981000 & 0.435000 & 3.961000\end{array}$

$\mathrm{H} \quad-3.788000 \quad 4.285000 \quad-0.319000$

$\begin{array}{llll}\text { C } & -5.779000 & 4.512000 & -0.789000\end{array}$

$\begin{array}{llll}\text { O } & -6.585000 & 4.917000 & -1.620000\end{array}$

$\begin{array}{llll}\mathrm{N} & -6.115000 & 4.245000 & 0.522000\end{array}$

$\mathrm{H} \quad-5.473000 \quad 3.688000 \quad 1.069000$

$\begin{array}{llll}\text { C } & -7.513000 & 4.253000 & 0.946000\end{array}$

$\begin{array}{llll}\text { C } & -8.284000 & 2.954000 & 0.669000\end{array}$

$\mathrm{H} \quad-7.530000 \quad 4.482000 \quad 2.019000$

$\begin{array}{llll}\mathrm{C} & -7.721000 & 1.718000 & 1.382000\end{array}$

$\begin{array}{llll}\mathrm{H} & -8.305000 & 2.784000 & -0.415000\end{array}$

$\begin{array}{llll}\mathrm{H} & -9.327000 & 3.110000 & 0.981000\end{array}$

$\mathrm{H} \quad-6.695000 \quad 1.532000 \quad 1.037000$

C $\quad-8.561000 \quad 0.457000 \quad 1.154000$

$\begin{array}{llll}\mathrm{H} & -7.652000 & 1.917000 & 2.463000\end{array}$

$\begin{array}{llll}\mathrm{H} & -8.120000 & -0.412000 & 1.657000\end{array}$

$\begin{array}{llll}H & -8.120000 & -0.412000 & 1.657000 \\ \mathrm{H} & -8.634000 & 0.219000 & 0.086000\end{array}$

$\begin{array}{llll}\mathrm{H} & -9.581000 & 0.586000 & 1.534000\end{array}$

$\mathrm{N} \quad-2.860000 \quad-3.209000 \quad-1.459000$

C $\quad-4.071000 \quad-2.760000 \quad-1.980000$

$\mathrm{H} \quad-2.176000 \quad-2.479000 \quad-1.276000$

$\mathrm{O} \quad-4.756000 \quad-3.436000 \quad-2.747000$

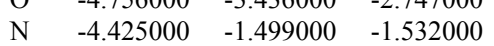

$\begin{array}{llll}\text { C } & -5.733000 & -0.952000 & -1.891000\end{array}$

$\mathrm{H} \quad-4.064000 \quad-1.242000 \quad-0.617000$

$\mathrm{H} \quad-6.244000 \quad-1.719000 \quad-2.479000$

$\begin{array}{llll}\text { C } & -5.660000 & 0.351000 & -2.697000\end{array}$

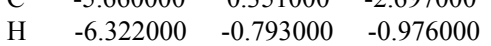

$\mathrm{H} \quad-4.987000 \quad 0.193000 \quad-3.551000$

$\begin{array}{llll}\mathrm{H} & -5.214000 & 1.150000 & -2.088000\end{array}$

$\begin{array}{llll}\text { C } & -7.041000 & 0.788000 & -3.206000\end{array}$

H $\quad-7.456000 \quad-0.015000 \quad-3.830000$

$\begin{array}{llll}\text { C } & -7.456000 & -0.015000 & -3.830000 \\ & -7.022000 & 2.099000 & -3.999000\end{array}$
C $\quad-3.891000 \quad 4.845000 \quad-2.317000$

$\begin{array}{llll}\mathrm{H} & -7.992000 & 5.084000 & 0.423000\end{array}$

\begin{tabular}{|c|c|c|c|}
\hline $\mathrm{H}$ & -7.729000 & 0.894000 & -2.353000 \\
\hline $\mathrm{H}$ & -6.330000 & 2.035000 & -4.848000 \\
\hline $\mathrm{H}$ & -6.722000 & 2.945000 & -3.371000 \\
\hline $\mathrm{H}$ & -8.015000 & 2.327000 & -4.401000 \\
\hline $\mathrm{H}$ & 7.901000 & 2.189000 & -3.768000 \\
\hline $\mathrm{H}$ & 8.277000 & 0.511000 & -3.345000 \\
\hline $\mathrm{H}$ & 7.900000 & 0.936000 & -5.014000 \\
\hline $\mathrm{H}$ & 7.031000 & -4.806000 & 0.023000 \\
\hline $\mathrm{H}$ & 7.503000 & -3.155000 & -0.381000 \\
\hline $\mathrm{H}$ & 6.766000 & -3.514000 & 1.192000 \\
\hline $\mathrm{H}$ & 6.520000 & 6.222000 & -1.545000 \\
\hline $\mathrm{H}$ & 6.506000 & 6.342000 & 0.222000 \\
\hline $\mathrm{H}$ & 5.492000 & 7.414000 & -0.743000 \\
\hline $\mathrm{H}$ & 6.416000 & 2.101000 & 3.937000 \\
\hline $\mathrm{H}$ & 6.033000 & 0.496000 & 4.589000 \\
\hline $\mathrm{H}$ & 5.739000 & 1.938000 & 5.557000 \\
\hline $\mathrm{H}$ & 2.364000 & -6.005000 & 5.156000 \\
\hline $\mathrm{H}$ & 2.359000 & -4.693000 & 6.347000 \\
\hline $\mathrm{H}$ & 3.812000 & -5.027000 & 5.399000 \\
\hline $\mathrm{H}$ & -4.351000 & 1.769000 & 2.299000 \\
\hline $\mathrm{H}$ & -4.023000 & 0.482000 & 5.054000 \\
\hline $\mathrm{H}$ & -2.990000 & 0.063000 & 3.681000 \\
\hline $\mathrm{N}$ & -4.985000 & -0.510000 & 000 \\
\hline $\mathrm{C}$ & -4.729000 & -1.302000 & 2.384000 \\
\hline $\mathrm{H}$ & -5.626000 & -0.869000 & 4.187000 \\
\hline $\mathrm{O}$ & -3.780000 & -1.057000 & 1.622000 \\
\hline $\mathrm{N}$ & -5.595000 & -2.340000 & 2.211000 \\
\hline $\mathrm{C}$ & -5.443000 & -3.381000 & 1.194000 \\
\hline $\mathrm{H}$ & -6.382000 & -2.429000 & 2.840000 \\
\hline $\mathrm{C}$ & -5.799000 & -4.757000 & 1.767000 \\
\hline $\mathrm{H}$ & -4.403000 & -3.361000 & 0.860000 \\
\hline $\mathrm{H}$ & -6.070000 & -3.154000 & 0.324000 \\
\hline $\mathrm{H}$ & -5.191000 & -4.929000 & 2.665000 \\
\hline $\mathrm{C}$ & -5.586000 & -5.914000 & 0.776000 \\
\hline $\mathrm{H}$ & -6.850000 & -4.754000 & 2.095000 \\
\hline $\mathrm{H}$ & -5.766000 & -6.853000 & 1.317000 \\
\hline $\mathrm{H}$ & -4.530000 & -5.935000 & 0.473000 \\
\hline $\mathrm{C}$ & -6.476000 & -5.868000 & -0.472000 \\
\hline $\mathrm{H}$ & -7.535000 & -5.770000 & -0.199000 \\
\hline $\mathrm{H}$ & -6.371000 & -6.7 & -1.051000 \\
\hline $\mathrm{H}$ & -6.216000 & -5.040000 & -1.140000 \\
\hline $\mathrm{C}$ & 5.698000 & 0.852000 & 0.447000 \\
\hline $\mathrm{C}$ & 4.618000 & -0.181000 & 0.752000 \\
\hline $\mathrm{N}$ & 3.242000 & 0.419000 & 0.603000 \\
\hline $\mathrm{H}$ & 4.664000 & -1.032000 & 0.071000 \\
\hline $\mathrm{H}$ & 4.677000 & -0.549000 & 1.779000 \\
\hline $\mathrm{H}$ & 2.471000 & -0.258000 & 0.783000 \\
\hline $\mathrm{H}$ & 3.110000 & 0.793000 & -0.342000 \\
\hline $\mathrm{H}$ & 3.100000 & 1.206000 & 1.253000 \\
\hline $\mathrm{H}$ & 5.604000 & 1.181000 & -0.596000 \\
\hline $\mathrm{H}$ & 5.549000 & 1.738000 & 1.077000 \\
\hline $\mathrm{C}$ & 7.106000 & 0.287000 & 0.681000 \\
\hline $\mathrm{H}$ & 7.245000 & -0.608000 & 0.060000 \\
\hline $\mathrm{H}$ & 7.199000 & -0.044000 & 1.724000 \\
\hline $\mathrm{C}$ & 8.206000 & 1.305000 & 0.365000 \\
\hline $\mathrm{H}$ & 9.199000 & 0.875000 & 0.533000 \\
\hline $\mathrm{H}$ & 8.118000 & 2.197000 & 0.997000 \\
\hline $\mathrm{H}$ & 8.154000 & 1.631000 & -0.680000 \\
\hline
\end{tabular}


Coordinates for $\left[\mathbf{2} \cdot \mathrm{H}^{+} \subset \mathbf{1} \supset \mathrm{Cl}^{-}\right]$

\begin{tabular}{|c|c|c|c|}
\hline $\mathrm{O}$ & 1.077000 & 1.190000 & 3.381000 \\
\hline $\mathrm{C}$ & 2.442000 & 1.140000 & 3.575000 \\
\hline $\mathrm{C}$ & 3.224000 & 2.285000 & 3.310000 \\
\hline $\mathrm{C}$ & 4.613000 & 2.187000 & 3.462000 \\
\hline $\mathrm{C}$ & 5.243000 & 0.996000 & 3.838000 \\
\hline $\mathrm{O}$ & 1.342000 & 2.706000 & 0.372000 \\
\hline $\mathrm{C}$ & 4.443000 & -0.138000 & 4.028000 \\
\hline $\mathrm{C}$ & 2.378000 & 3.626000 & 0.270000 \\
\hline $\mathrm{C}$ & 3.049000 & -0.095000 & 3.904000 \\
\hline $\mathrm{C}$ & 2.784000 & 4.050000 & -1.013000 \\
\hline $\mathrm{C}$ & 3.817000 & 4.994000 & -1.073000 \\
\hline $\mathrm{C}$ & 4.464000 & 5.484000 & 0.066000 \\
\hline $\mathrm{C}$ & 4.053000 & 5.005000 & 1.313000 \\
\hline $\mathrm{C}$ & 3.016000 & 4.074000 & 1.446000 \\
\hline $\mathrm{C}$ & 5.561000 & 6.517000 & -0.054000 \\
\hline $\mathrm{C}$ & 2.606000 & 3.604000 & 2.846000 \\
\hline $\mathrm{C}$ & 6.738000 & 0.933000 & 4.046000 \\
\hline $\mathrm{C}$ & 2.101000 & 3.555000 & -2.292000 \\
\hline $\mathrm{C}$ & 2.216000 & -1.360000 & 4.107000 \\
\hline $\mathrm{O}$ & 1.125000 & 0.897000 & -3.022000 \\
\hline $\mathrm{C}$ & 2.412000 & 1.241000 & -3.396000 \\
\hline $\mathrm{C}$ & 3.182000 & 0.322000 & -4.150000 \\
\hline $\mathrm{C}$ & 4.367000 & 0.779000 & -4.737000 \\
\hline $\mathrm{C}$ & 4.838000 & 2.082000 & -4.554000 \\
\hline $\mathrm{C}$ & 4.097000 & 2.932000 & -3.731000 \\
\hline $\mathrm{C}$ & 2.891000 & 2.544000 & -3.132000 \\
\hline $\mathrm{C}$ & 6.114000 & 2.553000 & -5.213000 \\
\hline $\mathrm{C}$ & 2.780000 & -1.141000 & -4.323000 \\
\hline $\mathrm{O}$ & 1.298000 & -3.061000 & -2.709000 \\
\hline $\mathrm{C}$ & 2.632000 & -2.846000 & -2.416000 \\
\hline $\mathrm{C}$ & 3.178000 & -3.430000 & -1.253000 \\
\hline $\mathrm{C}$ & 4.564000 & -3.384000 & -1.078000 \\
\hline $\mathrm{C}$ & 5.404000 & -2.759000 & -2.008000 \\
\hline $\mathrm{C}$ & 4.809000 & -2.052000 & -3.058000 \\
\hline $\mathrm{C}$ & 3.423000 & -2.046000 & -3.264000 \\
\hline $\mathrm{C}$ & 6.907000 & -2.838000 & -1.873000 \\
\hline $\mathrm{O}$ & 1.498000 & -1.793000 & 1.343000 \\
\hline $\mathrm{C}$ & 2.272000 & -2.770000 & 1.966000 \\
\hline $\mathrm{C}$ & 2.655000 & -2.583000 & 3.306000 \\
\hline $\mathrm{C}$ & 3.436000 & -3.578000 & 3.907000 \\
\hline $\mathrm{C}$ & 3.843000 & -4.722000 & 3.213000 \\
\hline $\mathrm{C}$ & 3.448000 & -4.860000 & 1.878000 \\
\hline $\mathrm{C}$ & 2.668000 & -3.899000 & 1.225000 \\
\hline $\mathrm{C}$ & 4.693000 & -5.777000 & 3.883000 \\
\hline $\mathrm{C}$ & 2.256000 & -4.092000 & -0.228000 \\
\hline $\mathrm{H}$ & 5.220000 & 3.067000 & 3.263000 \\
\hline $\mathrm{H}$ & 4.917000 & -1.087000 & 4.268000 \\
\hline $\mathrm{H}$ & 4.106000 & 5.393000 & -2.042000 \\
\hline $\mathrm{H}$ & 4.532000 & 5.389000 & 2.211000 \\
\hline $\mathrm{H}$ & 2.912000 & 4.385000 & 3.552000 \\
\hline $\mathrm{H}$ & 1.524000 & 3.526000 & 2.923000 \\
\hline $\mathrm{H}$ & 1.913000 & 4.436000 & -2.920000 \\
\hline $\mathrm{H}$ & 1.122000 & 3.151000 & -2.044000 \\
\hline $\mathrm{H}$ & 2.247000 & -1.628000 & 5.170000 \\
\hline $\mathrm{H}$ & 1.178000 & -1.122000 & 3.880000 \\
\hline $\mathrm{H}$ & 4.929000 & 0.100000 & -5.377000 \\
\hline $\mathrm{H}$ & 4.455000 & 3.945000 & -3.577000 \\
\hline $\mathrm{H}$ & 3.080000 & -1.470000 & -5.326000 \\
\hline $\mathrm{H}$ & 1.696000 & -1.243000 & -4.263000 \\
\hline $\mathrm{H}$ & 5.000000 & -3.859000 & -0.202000 \\
\hline $\mathrm{H}$ & 5.444000 & -1.483000 & -3.733000 \\
\hline $\mathrm{H}$ & 3.713000 & -3.467000 & 4.953000 \\
\hline $\mathrm{H}$ & 3.741000 & -5.754000 & 1.330000 \\
\hline $\mathrm{H}$ & 2.205000 & -5.172000 & -0.425000 \\
\hline $\mathrm{H}$ & 1.251000 & -3.698000 & -0.394000 \\
\hline $\mathrm{C}$ & 1.102000 & -4.213000 & -3.571000 \\
\hline $\mathrm{C}$ & 0.253000 & 1.404000 & 4.548000 \\
\hline $\mathrm{C}$ & 0.058000 & -1.957000 & 1.493000 \\
\hline $\mathrm{C}$ & 0.971000 & 0.159000 & -1.805000 \\
\hline $\mathrm{C}$ & 0.082000 & 3.220000 & 0.908000 \\
\hline $\mathrm{C}$ & -1.082000 & 3.069000 & -0.043000 \\
\hline $\mathrm{H}$ & 0.216000 & 4.277000 & 1.156000 \\
\hline $\mathrm{H}$ & -0.126000 & 2.652000 & 1.816000 \\
\hline $\mathrm{H}$ & 0.314000 & 2.453000 & 4.866000 \\
\hline
\end{tabular}

$\begin{array}{rrrrr}\mathrm{H} & 0.590000 & 0.771000 & 5.377000 & \mathrm{H} \\ \mathrm{C} & -1.170000 & 1.042000 & 4.139000 & \mathrm{H} \\ \mathrm{H} & -1.214000 & -0.007000 & 3.822000 & \mathrm{H} \\ \mathrm{O} & -2.004000 & 1.291000 & 5.248000 & \mathrm{H} \\ \mathrm{H} & -1.994000 & 3.251000 & 0.541000 & \mathrm{H} \\ \mathrm{O} & -0.946000 & 3.987000 & -1.114000 & \mathrm{H} \\ \mathrm{H} & 1.701000 & -0.652000 & -1.755000 & \mathrm{H} \\ \mathrm{C} & -0.422000 & -0.466000 & -1.774000 & \mathrm{H} \\ \mathrm{H} & 1.115000 & 0.840000 & -0.961000 & \mathrm{H} \\ \mathrm{O} & -0.485000 & -1.456000 & -0.758000 & \mathrm{H} \\ \mathrm{H} & -0.596000 & -0.982000 & -2.718000 & \mathrm{H} \\ \mathrm{H} & -1.209000 & 0.286000 & -1.645000 & \mathrm{H} \\ \mathrm{H} & -0.225000 & -1.776000 & 2.534000 & \mathrm{H} \\ \mathrm{H} & -0.213000 & -2.986000 & 1.236000 & \mathrm{H}\end{array}$

$\begin{array}{llll}\mathrm{H} & -0.213000 & -2.986000 & 1.236000\end{array}$

$\begin{array}{llll}\text { C } & -0.643000 & -0.985000 & 0.577000\end{array}$

$\begin{array}{llrr}\mathrm{H} & -0.209000 & 0.014000 & 0.713000\end{array}$

$\begin{array}{llll}\mathrm{H} & -1.704000 & -0.927000 & 0.843000\end{array}$

C $\quad-0.185000 \quad-4.978000 \quad-3.239000$

$\begin{array}{llll}\mathrm{H} & 1.957000 & -4.887000 & -3.437000\end{array}$

$\begin{array}{llll}\mathrm{H} & 1.080000 & -3.887000 & -4.619000\end{array}$

O $\quad-1.318000 \quad-4.634000 \quad-4.005000$

$\mathrm{H} \quad-0.367000 \quad-4.913000 \quad-2.158000$

$\mathrm{H} \quad-0.003000 \quad-6.033000 \quad-3.479000$

C $\quad-2.049000 \quad-3.466000 \quad-3.629000$

$\mathrm{H} \quad-2.846000 \quad-3.385000 \quad-4.372000$

H $\quad-1.416000 \quad-2.570000 \quad-3.701000$

$\begin{array}{llll}\text { C } & -2.694000 & -3.566000 & -2.238000\end{array}$

$\mathrm{H} \quad-1.933000 \quad-3.517000 \quad-1.455000$

$\begin{array}{llll}\mathrm{H} & -3.216000 & -4.525000 & -2.176000\end{array}$

$\begin{array}{llll}\mathrm{H} & -1.147000 & 2.039000 & -0.415000\end{array}$

$\begin{array}{llll}\mathrm{H} & -1.476000 & 1.653000 & 3.275000\end{array}$

$\begin{array}{llll}\text { C } & -3.393000 & 1.401000 & 4.944000\end{array}$

C $\quad-1.847000 \quad 3.786000 \quad-2.203000$

$\mathrm{H} \quad-1.906000 \quad 2.713000 \quad-2.445000$

$\mathrm{H} \quad-1.385000 \quad 4.291000 \quad-3.060000$

C $\quad-3.248000 \quad 4.367000 \quad-1.994000$

$\mathrm{H} \quad-3.182000 \quad 5.421000 \quad-1.713000$

$\mathrm{N} \quad-4.007000 \quad 3.704000 \quad-0.956000$

$\begin{array}{llll}\mathrm{H} & -3.771000 & 4.313000 & -2.963000\end{array}$

$\mathrm{H} \quad-3.839000 \quad 1.884000 \quad 5.820000$

$\begin{array}{llll}\mathrm{C} & -4.114000 & 0.075000 & 4.704000\end{array}$

$\begin{array}{llll}\mathrm{H} & -4.022000 & 2.687000 & -0.926000\end{array}$

$\begin{array}{llll}\text { C } & -4.482000 & 4.399000 & 0.139000\end{array}$

$\begin{array}{llll}\mathrm{O} & -4.498000 & 5.629000 & 0.201000\end{array}$

$\begin{array}{llll}\mathrm{N} & -4.905000 & 3.587000 & 1.173000\end{array}$

$\begin{array}{llll}\mathrm{H} & -4.925000 & 2.584000 & 1.007000\end{array}$

$\begin{array}{llll}\text { C } & -5.723000 & 4.134000 & 2.245000\end{array}$

$\begin{array}{llll}\text { C } & -7.233000 & 4.138000 & 1.958000\end{array}$

$\mathrm{H} \quad-5.377000 \quad 5.157000 \quad 2.411000$

$\mathrm{H} \quad-5.518000 \quad 3.559000 \quad 3.159000$

$\begin{array}{llll}\mathrm{C} & -7.847000 & 2.744000 & 1.789000\end{array}$

$\mathrm{H} \quad-7.409000 \quad 4.737000 \quad 1.053000$

$\mathrm{H} \quad-7.739000 \quad 4.662000 \quad 2.783000$

$\mathrm{H} \quad-7.324000 \quad 2.208000 \quad 0.985000$

$\begin{array}{llll}\text { C } & -9.347000 & 2.779000 & 1.479000\end{array}$

$\mathrm{H} \quad-7.678000 \quad 2.159000 \quad 2.706000$

$\begin{array}{llll}\mathrm{H} & -9.755000 & 1.767000 & 1.372000\end{array}$

$\begin{array}{llll}\mathrm{H} & -9.546000 & 3.318000 & 0.545000\end{array}$

$\mathrm{H} \quad-9.907000 \quad 3.282000 \quad 2.277000$

$\begin{array}{llll}\mathrm{N} & -3.638000 & -2.490000 & -1.996000\end{array}$

C $\quad-4.960000 \quad-2.631000 \quad-2.375000$

$\mathrm{H} \quad-3.381000 \quad-1.730000 \quad-1.378000$

O $\quad-5.341000 \quad-3.577000 \quad-3.074000$

$\mathrm{N} \quad-5.802000 \quad-1.660000 \quad-1.896000$

$\begin{array}{llll}\mathrm{C} & -7.172000 & -1.562000 & -2.371000\end{array}$

$\mathrm{H} \quad-5.408000 \quad-0.880000 \quad-1.374000$

$\begin{array}{llll}\mathrm{H} & -7.530000 & -2.581000 & -2.542000\end{array}$

$\begin{array}{llll}\text { C } & -7.325000 & -0.736000 & -3.657000\end{array}$

H $\quad-7.777000 \quad-1.125000 \quad-1.567000$

$\mathrm{H} \quad-6.704000 \quad-1.195000 \quad-4.437000$

$\mathrm{H} \quad-6.926000 \quad 0.275000 \quad-3.488000$

C $\quad-8.778000 \quad-0.643000 \quad-4.136000$

H $\quad-9.171000 \quad-1.657000 \quad-4.298000$

$\begin{array}{llll}\mathrm{H} & -9.171000 & -1.657000 & -4.298000 \\ \mathrm{C} & -8.937000 & 0.175000 & -5.422000\end{array}$
H $\quad-9.397000 \quad-0.200000 \quad-3.343000$

$\begin{array}{llll}\mathrm{H} & -8.356000 & -0.263000 & -6.243000\end{array}$

$\mathrm{H} \quad-8.588000 \quad 1.205000 \quad-5.283000$

$\begin{array}{llll}\mathrm{H} & -9.985000 & 0.219000 & -5.743000\end{array}$

$\mathrm{H} \quad 6.967000 \quad 2.511000 \quad-4.522000$

$\begin{array}{llll}\mathrm{H} & 6.368000 & 1.935000 & -6.080000\end{array}$

$\mathrm{H} \quad 6.029000 \quad 3.591000 \quad-5.555000$

H $\quad 7.302000 \quad-3.732000 \quad-2.376000$

$\begin{array}{llll}\mathrm{H} & 7.402000 & -1.971000 & -2.325000\end{array}$

$\begin{array}{llll}\mathrm{H} & 7.218000 & -2.898000 & -0.824000\end{array}$

$\begin{array}{llll}\mathrm{H} & 6.438000 & 6.116000 & -0.577000\end{array}$

$\mathrm{H} \quad 5.892000 \quad 6.865000 \quad 0.929000$

$\begin{array}{llll}\mathrm{H} & 5.222000 & 7.393000 & -0.621000\end{array}$

$\begin{array}{llll}\mathrm{H} & 7.000000 & 1.160000 & 5.088000\end{array}$

$\begin{array}{llll}\mathrm{H} & 7.266000 & 1.656000 & 3.415000\end{array}$

$\begin{array}{llll}\mathrm{H} & 7.132000 & -0.065000 & 3.824000\end{array}$

$\mathrm{H} \quad 4.424000 \quad-6.783000 \quad 3.541000$

$\begin{array}{llll}\mathrm{H} & 4.580000 & -5.750000 & 4.971000\end{array}$

H $\quad 5.758000 \quad-5.634000 \quad 3.660000$

$\begin{array}{llll}\mathrm{H} & -3.531000 & 2.074000 & 4.081000\end{array}$

$\begin{array}{llll}\mathrm{H} & -5.198000 & 0.272000 & 4.777000\end{array}$

$\mathrm{H} \quad-3.862000 \quad-0.637000 \quad 5.494000$

$\begin{array}{llll}\mathrm{N} & -3.772000 & -0.552000 & 3.438000\end{array}$

$\begin{array}{llll}\text { C } & -3.801000 & -1.938000 & 3.331000\end{array}$

$\mathrm{H} \quad-3.981000 \quad-0.032000 \quad 2.587000$

$\begin{array}{llll}\mathrm{O} & -3.816000 & -2.682000 & 4.311000\end{array}$

$\begin{array}{llll}\mathrm{N} & -3.728000 & -2.395000 & 2.029000\end{array}$

$\begin{array}{llll}\text { C } & -4.051000 & -3.787000 & 1.726000\end{array}$

$\begin{array}{llll}\mathrm{H} & -3.947000 & -1.715000 & 1.303000\end{array}$

$\begin{array}{llll}\text { C } & -5.547000 & -4.022000 & 1.470000\end{array}$

$\begin{array}{llll}\mathrm{H} & -3.714000 & -4.383000 & 2.579000\end{array}$

$\mathrm{H} \quad-3.464000 \quad-4.086000 \quad 0.851000$

$\begin{array}{llll}\mathrm{H} & -6.096000 & -3.699000 & 2.365000\end{array}$

$\begin{array}{llll}\text { C } & -5.907000 & -5.482000 & 1.149000\end{array}$

$\mathrm{H} \quad-5.874000 \quad-3.375000 \quad 0.643000$

$\begin{array}{llll}\mathrm{H} & -7.002000 & -5.567000 & 1.118000\end{array}$

$\begin{array}{llll}\mathrm{H} & -5.577000 & -6.128000 & 1.977000\end{array}$

$\begin{array}{llll}\text { C } & -5.333000 & -6.005000 & -0.175000\end{array}$

$\begin{array}{llll}\mathrm{H} & -5.612000 & -5.362000 & -1.018000\end{array}$

$\begin{array}{llll}\mathrm{H} & -5.700000 & -7.017000 & -0.387000\end{array}$

$\begin{array}{llll}\mathrm{H} & -4.237000 & -6.059000 & -0.151000\end{array}$

C $\quad 5.434000 \quad 1.328000 \quad-0.124000$

$\begin{array}{llll}\mathrm{C} & 4.390000 & 0.231000 & -0.314000\end{array}$

$\begin{array}{lllr}\mathrm{N} & 3.267000 & 0.337000 & 0.682000\end{array}$

$\mathrm{H} \quad 3.939000 \quad 0.273000 \quad-1.305000$

$\begin{array}{llll}\mathrm{H} & 4.818000 & -0.764000 & -0.182000\end{array}$

$\mathrm{H} \quad 2.609000 \quad-0.469000 \quad 0.656000$

$\begin{array}{llll}\mathrm{H} & 2.689000 & 1.186000 & 0.565000\end{array}$

$\begin{array}{llll}\mathrm{H} & 3.632000 & 0.359000 & 1.640000\end{array}$

$\mathrm{H} \quad 4.961000 \quad 2.312000 \quad-0.224000$

$\begin{array}{llll}\mathrm{H} & 5.844000 & 1.274000 & 0.894000\end{array}$

$\begin{array}{llll}\text { C } & 6.573000 & 1.205000 & -1.144000\end{array}$

$\begin{array}{llll}\mathrm{H} & 6.152000 & 1.235000 & -2.156000\end{array}$

$\begin{array}{llll}\mathrm{H} & 7.060000 & 0.226000 & -1.036000\end{array}$

C $\quad 7.612000 \quad 2.319000 \quad-0.987000$

$\begin{array}{llll}\mathrm{H} & 8.419000 & 2.216000 & -1.720000\end{array}$

$\begin{array}{llll}\mathrm{H} & 8.066000 & 2.305000 & 0.012000\end{array}$

$\begin{array}{llll}\mathrm{H} & 7.153000 & 3.305000 & -1.129000\end{array}$

$\begin{array}{llll}\mathrm{Cl} & -4.034000 & 0.441000 & 0.106000\end{array}$ 
Coordinates for $\left[\mathbf{3} \cdot \mathrm{H}^{+} \subset \mathbf{1}\right]$

$\begin{array}{lrrr}\mathrm{H} & -0.328000 & 2.274000 & 4.471000 \\ \mathrm{C} & -1.763000 & 2.721000 & 2.935000 \\ \mathrm{H} & -1.842000 & 1.742000 & 2.442000 \\ \mathrm{O} & -2.694000 & 2.847000 & 3.991000 \\ \mathrm{H} & -0.786000 & 2.274000 & -2.297000 \\ \mathrm{O} & -1.988000 & 3.744000 & -1.444000 \\ \mathrm{H} & 1.444000 & -1.439000 & -1.321000 \\ \mathrm{C} & -0.562000 & -0.875000 & -1.804000\end{array}$

$\mathrm{H} \quad 1.122000 \quad 0.319000 \quad-1.151000$

$\begin{array}{llll}\mathrm{O} & -0.996000 & -1.497000 & -0.601000\end{array}$

$\mathrm{H} \quad-0.749000 \quad-1.595000 \quad-2.601000$

$\begin{array}{llll}\mathrm{H} & -1.147000 & 0.030000 & -2.019000\end{array}$

$\begin{array}{llll}\mathrm{H} & -0.870000 & -0.961000 & 2.677000\end{array}$

$\begin{array}{llll}\mathrm{H} & -0.847000 & -2.460000 & 1.730000\end{array}$

$\begin{array}{llll}\mathrm{C} & -1.080000 & -0.673000 & 0.563000\end{array}$

$\begin{array}{llrr}\mathrm{H} & -0.540000 & 0.271000 & 0.430000\end{array}$

$\begin{array}{llll}\mathrm{H} & -2.135000 & -0.443000 & 0.773000\end{array}$

$\begin{array}{llll}\text { C } & -0.312000 & -5.957000 & -1.746000\end{array}$

$\mathrm{H} \quad 1.834000 \quad-5.843000 \quad-1.926000$

H $\quad 0.951000 \quad-5.054000 \quad-3.251000$

$\begin{array}{llll}\mathrm{O} & -1.429000 & -5.746000 & -2.583000\end{array}$

$\begin{array}{llll}\mathrm{H} & -0.526000 & -5.728000 & -0.691000\end{array}$

$\mathrm{H} \quad-0.114000 \quad-7.033000 \quad-1.816000$

C $\quad-2.042000 \quad-4.465000 \quad-2.492000$

$\mathrm{H} \quad-2.618000 \quad-4.339000 \quad-3.413000$

$\begin{array}{llll}\mathrm{H} & -1.278000 & -3.677000 & -2.446000\end{array}$

$\begin{array}{llll}\text { C } & -2.996000 & -4.344000 & -1.295000\end{array}$

$\mathrm{H} \quad-2.474000 \quad-4.566000 \quad-0.358000$

$\mathrm{H} \quad-3.810000 \quad-5.063000 \quad-1.411000$

$\begin{array}{llll}\mathrm{H} & -0.194000 & 3.945000 & -2.478000\end{array}$

$\begin{array}{llll}\mathrm{H} & -1.936000 & 3.491000 & 2.167000\end{array}$

$\begin{array}{llll}\text { C } & -3.976000 & 2.296000 & 3.689000\end{array}$

C $\quad-2.880000 \quad 3.962000 \quad-2.536000$

$\mathrm{H} \quad-3.009000 \quad 3.031000 \quad-3.108000$

$\begin{array}{llll}\mathrm{H} & -2.476000 & 4.732000 & -3.211000\end{array}$

C $\quad-4.201000 \quad 4.421000 \quad-1.946000$

$\mathrm{H} \quad-4.062000 \quad 5.384000 \quad-1.434000$

$\mathrm{N} \quad \begin{array}{llll}-4.713000 & 3.398000 & -1.042000\end{array}$

$\begin{array}{llll}\mathrm{H} & -4.924000 & 4.584000 & -2.749000\end{array}$

$\begin{array}{llll}\mathrm{H} & -4.696000 & 2.808000 & 4.336000\end{array}$

$\mathrm{H} \quad-3.991000 \quad 2.959000 \quad-0.483000$

$\begin{array}{llll}\text { C } & -5.909000 & 3.669000 & -0.380000\end{array}$

$\begin{array}{llll}\mathrm{O} & -6.629000 & 4.613000 & -0.690000\end{array}$

$\mathrm{N} \quad-6.201000 \quad 2.793000 \quad 0.643000$

$\begin{array}{llll}\mathrm{H} & -5.740000 & 1.890000 & 0.662000\end{array}$

$\begin{array}{llll}\text { C } & -7.426000 & 2.968000 & 1.421000\end{array}$

$\begin{array}{llll}\text { C } & -8.727000 & 2.542000 & 0.726000\end{array}$

$\begin{array}{llll}\mathrm{H} & -7.493000 & 4.029000 & 1.679000\end{array}$

$\begin{array}{llll} & -7.290000 & 2.408000 & 2.355000\end{array}$

C $\quad-8.830000 \quad 1.046000 \quad 0.417000$

H $\quad-8.832000 \quad 3.126000 \quad-0.196000$

$\begin{array}{llll}H & -9.562000 & 2.836000 & 1.379000\end{array}$

$\begin{array}{llll}H & -8.012000 & 0.756000 & -0.257000\end{array}$

$\begin{array}{llll}\text { C } & -10.170000 & 0.658000 & -0.218000\end{array}$

$\begin{array}{llll}\mathrm{H} & -8.684000 & 0.471000 & 1.345000\end{array}$

H $\quad-10.222000 \quad-0.417000 \quad-0.426000$

$\begin{array}{llll}\mathrm{H} & -10.326000 & 1.189000 & -1.165000\end{array}$

H $\quad-11.008000 \quad 0.910000 \quad 0.442000$

$\mathrm{N} \quad-3.572000-3.010000-1.185000$

$\begin{array}{llll}\text { C } & -4.593000 & -2.652000 & -2.058000\end{array}$

$\begin{array}{llll}\mathrm{H} & -2.896000 & -2.287000 & -0.951000\end{array}$

$\begin{array}{llll}\mathrm{O} & -5.176000 & -3.473000 & -2.764000\end{array}$

N $\quad-4.893000 \quad-1.302000 \quad-2.047000$

C $\quad-6.073000 \quad-0.822000 \quad-2.764000$

$\mathrm{H} \quad-4.663000 \quad-0.801000 \quad-1.195000$

$\begin{array}{llll}\mathrm{H} & -6.231000 & -1.504000 & -3.602000\end{array}$

C $\quad-5.903000 \quad 0.619000 \quad-3.247000$

$\mathrm{H} \quad-6.971000 \quad-0.893000 \quad-2.128000$

$\begin{array}{llll}\mathrm{H} & -5.038000 & 0.667000 & -3.924000\end{array}$

$\mathrm{H} \quad-5.664000 \quad 1.272000 \quad-2.396000$

C $\quad-7.155000 \quad 1.151000 \quad-3.957000$

$\begin{array}{llll}\mathrm{H} & -7.341000 & 0.555000 & -4.862000\end{array}$

$\begin{array}{llll}\mathrm{C} & -7.058000 & 2.635000 & -4.326000\end{array}$
$\begin{array}{llll}\mathrm{C} & -3.971000 & 0.788000 & 3.978000\end{array}$

\begin{tabular}{|c|c|c|c|}
\hline $\mathrm{H}$ & -8.030000 & 0.998000 & -3.309000 \\
\hline $\mathrm{H}$ & -6.179000 & 2.831000 & -4.953000 \\
\hline $\mathrm{H}$ & -6.983000 & 3.261000 & -3.429000 \\
\hline $\mathrm{H}$ & -7.941000 & 2.963000 & -4.885000 \\
\hline $\mathrm{H}$ & 6.944000 & 1.362000 & -5.110000 \\
\hline $\mathrm{H}$ & 7.551000 & 0.219000 & -3.910000 \\
\hline $\mathrm{H}$ & 7.025000 & -0.364000 & -5.492000 \\
\hline $\mathrm{H}$ & 6.936000 & -4.862000 & -0.150000 \\
\hline $\mathrm{H}$ & 7.185000 & -3.116000 & -0.229000 \\
\hline $\mathrm{H}$ & 6.657000 & -3.872000 & 1.283000 \\
\hline $\mathrm{H}$ & 6.185000 & 5.742000 & -2.678000 \\
\hline $\mathrm{H}$ & 6.190000 & 6.360000 & -1.017000 \\
\hline $\mathrm{H}$ & 5.117000 & 7.072000 & -2.224000 \\
\hline $\mathrm{H}$ & 6.594000 & 2.776000 & 3.814000 \\
\hline $\mathrm{H}$ & 6.475000 & 1.020000 & 4.041000 \\
\hline $\mathrm{H}$ & 6.012000 & 2.113000 & 5.343000 \\
\hline $\mathrm{H}$ & 3.075000 & -5.324000 & 6.034000 \\
\hline $\mathrm{H}$ & 2.891000 & -3.898000 & 7.069000 \\
\hline $\mathrm{H}$ & 4.361000 & -4.116000 & 6.118000 \\
\hline $\mathrm{H}$ & -4.261000 & 2.488000 & 2.648000 \\
\hline $\mathrm{H}$ & -3.817000 & 0.637000 & 5.051000 \\
\hline $\mathrm{H}$ & -3.127000 & 0.323000 & 3.455000 \\
\hline $\mathrm{N}$ & -5.206000 & 0.109000 & 3.596000 \\
\hline $\mathrm{C}$ & -5.331000 & -0.452000 & 2.334000 \\
\hline $\mathrm{H}$ & -5.696000 & -0.355000 & 4.349000 \\
\hline $\mathrm{O}$ & -4.608000 & -0.089000 & 1.388000 \\
\hline $\mathrm{N}$ & -6.293000 & -1.409000 & 2.221000 \\
\hline $\mathrm{C}$ & -6.598000 & -2.111000 & 0.973000 \\
\hline $\mathrm{H}$ & -6.920000 & -1.568000 & 2.998000 \\
\hline $\mathrm{C}$ & -7.266000 & -3.458000 & 1.262000 \\
\hline $\mathrm{H}$ & -5.654000 & -2.270000 & 0.449000 \\
\hline $\mathrm{H}$ & -7.236000 & -1.486000 & 0.338000 \\
\hline $\mathrm{H}$ & -6.559000 & -4.080000 & 1.826000 \\
\hline $\mathrm{C}$ & -7.717000 & -4.204000 & -0.007000 \\
\hline $\mathrm{H}$ & -8.139000 & -3.303000 & 1.917000 \\
\hline $\mathrm{H}$ & -7.939000 & -5.242000 & 0.271000 \\
\hline $\mathrm{H}$ & -6.889000 & -4.249000 & -0.726000 \\
\hline $\mathrm{C}$ & -8.951000 & -3.601000 & -0.690000 \\
\hline $\mathrm{H}$ & -9.812000 & -3.581000 & -0.010000 \\
\hline $\mathrm{H}$ & -9.232000 & -4.193000 & -1.567000 \\
\hline $\mathrm{H}$ & -8.773000 & -2.577000 & -1.036000 \\
\hline $\mathrm{C}$ & 5.587000 & 0.882000 & -0.018000 \\
\hline $\mathrm{C}$ & 4.677000 & -0.151000 & 0.657000 \\
\hline $\mathrm{N}$ & 3.232000 & 0.286000 & 0.560000 \\
\hline $\mathrm{H}$ & 4.741000 & -1.128000 & 0.174000 \\
\hline $\mathrm{H}$ & 4.896000 & -0.261000 & 1.720000 \\
\hline $\mathrm{H}$ & 2.553000 & -0.396000 & 0.947000 \\
\hline $\mathrm{H}$ & 2.970000 & 0.443000 & -0.420000 \\
\hline $\mathrm{H}$ & 3.066000 & 1.175000 & 1.056000 \\
\hline $\mathrm{H}$ & 5.279000 & 0.998000 & -1.064000 \\
\hline $\mathrm{H}$ & 5.450000 & 1.855000 & 0.467000 \\
\hline $\mathrm{C}$ & 7.048000 & 0.478000 & 0.038000 \\
\hline $\mathrm{C}$ & 9.758000 & -0.271000 & 0.124000 \\
\hline $\mathrm{C}$ & 7.893000 & 0.966000 & 1.042000 \\
\hline $\mathrm{C}$ & 7.581000 & -0.389000 & -0.925000 \\
\hline $\mathrm{C}$ & 8.926000 & -0.760000 & -0.885000 \\
\hline $\mathrm{C}$ & 9.238000 & 0.593000 & 1.088000 \\
\hline $\mathrm{H}$ & 7.502000 & 1.655000 & 1.785000 \\
\hline $\mathrm{H}$ & 6.938000 & -0.765000 & -1.718000 \\
\hline $\mathrm{H}$ & 9.326000 & -1.426000 & -1.645000 \\
\hline $\mathrm{H}$ & 9.880000 & 0.986000 & 1.871000 \\
\hline $\mathrm{H}$ & 10.806000 & -0.556000 & 0.154000 \\
\hline
\end{tabular}


Coordinates for $\left[\mathbf{3} \cdot \mathrm{H}^{+} \subset \mathbf{1} \supset \mathrm{Cl}^{-}\right]$

\begin{tabular}{|c|c|c|c|}
\hline $\mathrm{O}$ & 0.796000 & 1.366000 & 3.213000 \\
\hline $\mathrm{C}$ & 2.158000 & 1.447000 & 3.403000 \\
\hline $\mathrm{C}$ & 2.854000 & 2.596000 & 2.966000 \\
\hline $\mathrm{C}$ & 4.238000 & 2.649000 & 3.176000 \\
\hline $\mathrm{C}$ & 4.944000 & 1.600000 & 3.777000 \\
\hline $\mathrm{O}$ & 0.889000 & 2.562000 & -0.067000 \\
\hline $\mathrm{C}$ & 4.235000 & 0.447000 & 4.129000 \\
\hline $\mathrm{C}$ & 1.971000 & 3.404000 & -0.273000 \\
\hline $\mathrm{C}$ & 2.849000 & 0.346000 & 3.958000 \\
\hline $\mathrm{C}$ & 2.474000 & 3.583000 & -1.579000 \\
\hline $\mathrm{C}$ & 3.553000 & 4.465000 & -1.743000 \\
\hline $\mathrm{C}$ & 4.148000 & 5.136000 & -0.672000 \\
\hline $\mathrm{C}$ & 3.656000 & 4.885000 & 0.613000 \\
\hline $\mathrm{C}$ & 2.584000 & 4.019000 & 0.842000 \\
\hline $\mathrm{C}$ & 5.261000 & 6.132000 & -0.898000 \\
\hline $\mathrm{C}$ & 2.127000 & 3.750000 & 2.276000 \\
\hline $\mathrm{C}$ & 6.424000 & 1.726000 & 4.062000 \\
\hline $\mathrm{C}$ & 1.851000 & 2.901000 & -2.801000 \\
\hline $\mathrm{C}$ & 2.108000 & -0.917000 & 4.384000 \\
\hline $\mathrm{O}$ & 0.930000 & 0.171000 & -3.004000 \\
\hline $\mathrm{C}$ & 2.207000 & 0.444000 & -3.452000 \\
\hline $\mathrm{C}$ & 2.977000 & -0.590000 & -4.033000 \\
\hline $\mathrm{C}$ & 4.146000 & -0.235000 & -4.716000 \\
\hline $\mathrm{C}$ & 4.598000 & 1.086000 & -4.791000 \\
\hline $\mathrm{C}$ & 3.860000 & 2.067000 & -4.124000 \\
\hline $\mathrm{C}$ & 2.669000 & 1.778000 & -3.446000 \\
\hline $\mathrm{C}$ & 5.816000 & 1.454000 & -5.608000 \\
\hline $\mathrm{C}$ & 2.584000 & -2.060000 & -3.930000 \\
\hline $\mathrm{O}$ & 1.151000 & -3.648000 & -1.955000 \\
\hline $\mathrm{C}$ & 2.503000 & -3.433000 & -1.764000 \\
\hline $\mathrm{C}$ & 3.101000 & -3.857000 & -0.556000 \\
\hline $\mathrm{C}$ & 4.491000 & -3.784000 & -0.438000 \\
\hline $\mathrm{C}$ & 5.290000 & -3.275000 & -1.467000 \\
\hline $\mathrm{C}$ & 4.651000 & -2.725000 & -2.583000 \\
\hline $\mathrm{C}$ & 3.258000 & -2.766000 & -2.747000 \\
\hline $\mathrm{C}$ & 6.798000 & -3.298000 & -1.364000 \\
\hline $\mathrm{O}$ & 1.454000 & -1.816000 & 1.696000 \\
\hline $\mathrm{C}$ & 2.237000 & -2.653000 & 2.493000 \\
\hline $\mathrm{C}$ & 2.606000 & -2.227000 & 3.783000 \\
\hline $\mathrm{C}$ & 3.421000 & -3.074000 & 4.544000 \\
\hline $\mathrm{C}$ & 3.867000 & -4.309000 & 4.064000 \\
\hline $\mathrm{C}$ & 3.464000 & -4.699000 & 2.782000 \\
\hline $\mathrm{C}$ & 2.655000 & -3.892000 & 1.975000 \\
\hline $\mathrm{C}$ & 4.756000 & -5.196000 & 4.904000 \\
\hline $\mathrm{C}$ & 2.231000 & -4.368000 & 0.592000 \\
\hline $\mathrm{H}$ & 4.780000 & 3.537000 & 2.861000 \\
\hline $\mathrm{H}$ & 4.771000 & -0.396000 & 4.560000 \\
\hline $\mathrm{H}$ & 3.921000 & 4.662000 & -2.746000 \\
\hline $\mathrm{H}$ & 4.108000 & 5.397000 & 1.460000 \\
\hline $\mathrm{H}$ & 2.296000 & 4.663000 & 2.860000 \\
\hline $\mathrm{H}$ & 1.059000 & 3.547000 & 2.307000 \\
\hline $\mathrm{H}$ & 1.683000 & 3.674000 & -3.562000 \\
\hline $\mathrm{H}$ & 0.875000 & 2.499000 & -2.541000 \\
\hline $\mathrm{H}$ & 2.184000 & -1.007000 & 5.475000 \\
\hline $\mathrm{H}$ & 1.050000 & -0.780000 & 4.163000 \\
\hline $\mathrm{H}$ & 4.705000 & -1.013000 & -5.231000 \\
\hline $\mathrm{H}$ & 4.201000 & 3.097000 & -4.175000 \\
\hline $\mathrm{H}$ & 2.868000 & -2.563000 & -4.863000 \\
\hline $\mathrm{H}$ & 1.501000 & -2.153000 & -3.833000 \\
\hline $\mathrm{H}$ & 4.961000 & -4.129000 & 0.479000 \\
\hline $\mathrm{H}$ & 5.257000 & -2.245000 & -3.345000 \\
\hline $\mathrm{H}$ & 3.692000 & -2.768000 & 5.552000 \\
\hline $\mathrm{H}$ & 3.774000 & -5.671000 & 2.401000 \\
\hline $\mathrm{H}$ & 2.237000 & -5.466000 & 0.599000 \\
\hline $\mathrm{H}$ & 1.203000 & -4.064000 & 0.385000 \\
\hline $\mathrm{C}$ & 0.852000 & -4.981000 & -2.444000 \\
\hline $\mathrm{C}$ & -0.012000 & 1.786000 & 4.335000 \\
\hline $\mathrm{C}$ & 0.015000 & -1.958000 & 1.897000 \\
\hline $\mathrm{C}$ & 0.790000 & -0.272000 & -1.651000 \\
\hline $\mathrm{C}$ & -0.401000 & 3.194000 & 0.137000 \\
\hline $\mathrm{C}$ & -1.045000 & 3.710000 & -1.148000 \\
\hline $\mathrm{H}$ & -0.303000 & 4.031000 & 0.836000 \\
\hline $\mathrm{H}$ & -1.042000 & 2.429000 & 0.579000 \\
\hline $\mathrm{H}$ & 0.105000 & 2.864000 & 4.502000 \\
\hline
\end{tabular}

$\begin{array}{rrrrr}\mathrm{H} & 0.302000 & 1.261000 & 5.245000 & \mathrm{H} \\ \mathrm{C} & -1.456000 & 1.438000 & 4.003000 & \mathrm{H} \\ \mathrm{H} & -1.539000 & 0.371000 & 3.761000 & \mathrm{H} \\ \mathrm{O} & -2.219000 & 1.776000 & 5.143000 & \mathrm{H} \\ \mathrm{H} & -1.269000 & 2.876000 & -1.831000 & \mathrm{H} \\ \mathrm{O} & -2.226000 & 4.353000 & -0.732000 & \mathrm{H} \\ \mathrm{H} & 1.400000 & -1.161000 & -1.471000 & \mathrm{H} \\ \mathrm{C} & -0.671000 & -0.636000 & -1.400000 & \mathrm{H} \\ \mathrm{H} & 1.087000 & 0.540000 & -0.981000 & \mathrm{H} \\ \mathrm{O} & -0.760000 & -1.563000 & -0.327000 & \mathrm{H} \\ \mathrm{H} & -1.064000 & -1.141000 & -2.284000 & \mathrm{H} \\ \mathrm{H} & -1.295000 & 0.248000 & -1.215000 & \mathrm{H} \\ \mathrm{H} & -0.222000 & -1.739000 & 2.942000 & \mathrm{H} \\ \mathrm{H} & -0.270000 & -2.994000 & 1.688000 & \mathrm{H}\end{array}$

$\begin{array}{llll}\mathrm{H} & -0.270000 & -2.994000 & 1.688000\end{array}$ $\begin{array}{lll}-0.736000 & -1.014000 & 0.988000\end{array}$

$\mathrm{H} \quad-0.262000 \quad-0.026000 \quad 1.017000$

$\begin{array}{llll}\mathrm{H} & -1.759000 & -0.897000 & 1.365000\end{array}$

$\begin{array}{llll}\text { C } & -0.555000 & -5.433000 & -2.046000\end{array}$

$\begin{array}{llll}\mathrm{H} & 1.585000 & -5.678000 & -2.017000\end{array}$

H $\quad 0.954000 \quad-5.002000 \quad-3.537000$

$\begin{array}{llll}\mathrm{O} & -1.572000 & -5.122000 & -2.972000\end{array}$

$\begin{array}{llll}\mathrm{H} & -0.774000 & -5.058000 & -1.036000\end{array}$

$\mathrm{H} \quad-0.541000 \quad-6.529000 \quad-1.996000$

C $\quad-2.091000 \quad-3.791000 \quad-2.919000$

$\mathrm{H} \quad-2.653000 \quad-3.656000 \quad-3.845000$

H $\quad-1.271000 \quad-3.064000 \quad-2.892000$

$\mathrm{H} \quad-2.520000 \quad-3.712000 \quad-0.787000$

$\mathrm{H} \quad-3.846000 \quad-4.323000 \quad-1.802000$

$\begin{array}{llll}\mathrm{H} & -0.375000 & 4.412000 & -1.673000\end{array}$

$\begin{array}{lllr}\mathrm{H} & -1.789000 & 2.000000 & 3.116000\end{array}$

$\begin{array}{llll}\text { C } & -3.627000 & 1.853000 & 4.930000\end{array}$

C $\quad-3.084000 \quad 4.766000 \quad-1.792000$

$\mathrm{H} \quad-3.187000 \quad 3.956000 \quad-2.530000$

$\mathrm{H} \quad-2.663000 \quad 5.650000 \quad-2.299000$

$\begin{array}{llll}\mathrm{C} & -4.438000 & 5.090000 & -1.188000\end{array}$

$\begin{array}{llll}\mathrm{H} & -4.313000 & 5.878000 & -0.429000\end{array}$

$\mathrm{N} \quad-5.028000 \quad 3.888000 \quad-0.636000$

$\mathrm{H} \quad-5.102000 \quad 5.493000 \quad-1.958000$

$\mathrm{H} \quad-4.015000 \quad 2.402000 \quad 5.795000$

$\begin{array}{llll}\mathrm{C} & -4.346000 & 0.509000 & 4.852000\end{array}$

$\mathrm{H} \quad-4.394000 \quad 3.204000 \quad-0.229000$

$\begin{array}{llll}\text { C } & -6.337000 & 3.911000 & -0.200000\end{array}$

$\begin{array}{llll}\mathrm{O} & -7.097000 & 4.851000 & -0.451000\end{array}$

$\begin{array}{llll}\mathrm{N} & -6.715000 & 2.794000 & 0.507000\end{array}$

$\begin{array}{llll}\mathrm{H} & -6.030000 & 2.058000 & 0.650000\end{array}$

$\begin{array}{llll}\text { C } & -8.031000 & 2.687000 & 1.113000\end{array}$

$\begin{array}{llll}\text { C } & -8.978000 & 1.699000 & 0.417000\end{array}$

$\begin{array}{llll}\mathrm{H} & -8.462000 & 3.691000 & 1.086000\end{array}$

$\mathrm{H} \quad-7.915000 \quad 2.400000 \quad 2.169000$

$\begin{array}{llll}\mathrm{C} & -8.538000 & 0.233000 & 0.507000\end{array}$

$\begin{array}{llll}\mathrm{H} & -9.081000 & 1.996000 & -0.635000\end{array}$

$\begin{array}{llll}\mathrm{H} & -9.975000 & 1.804000 & 0.873000\end{array}$

$\begin{array}{llll}\mathrm{H} & -7.543000 & 0.121000 & 0.055000\end{array}$

$\begin{array}{llll}\text { C } & -9.516000 & -0.730000 & -0.173000\end{array}$

$\mathrm{H} \quad-8.426000 \quad-0.048000 \quad 1.565000$

$\mathrm{H} \quad-9.172000 \quad-1.768000 \quad-0.100000$

$\begin{array}{llll}\mathrm{H} & -9.630000 & -0.493000 & -1.238000\end{array}$

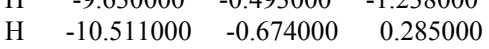

$\mathrm{N} \quad-3.624000 \quad-2.246000 \quad-1.725000$

C $\quad-4.704000 \quad-1.933000 \quad-2.532000$

$\mathrm{H} \quad-3.276000 \quad-1.563000 \quad-1.063000$

O $\quad-5.195000 \quad-2.758000 \quad-3.308000$

$\mathrm{N} \quad-5.145000 \quad-0.640000 \quad-2.411000$

$\begin{array}{llll}\text { C } & -6.350000 & -0.187000 & -3.087000\end{array}$

$\begin{array}{llll}\mathrm{H} & -4.806000 & -0.082000 & -1.630000\end{array}$

$\begin{array}{llll}\mathrm{H} & -6.425000 & -0.741000 & -4.027000\end{array}$

$\begin{array}{llll}\text { C } & -6.307000 & 1.324000 & -3.335000\end{array}$

$\mathrm{H} \quad-7.250000 \quad-0.438000 \quad-2.501000$

$\begin{array}{llll}\mathrm{H} & -5.449000 & 1.557000 & -3.981000\end{array}$

$\mathrm{H} \quad-6.128000 \quad 1.845000 \quad-2.385000$

C $\quad-7.597000 \quad 1.862000 \quad-3.966000$

$\mathrm{H} \quad-7.753000 \quad 1.385000 \quad-4.944000$ $\begin{array}{llll}\text { C } & -3.048000 & -3.577000 & -1.735000\end{array}$

\begin{tabular}{|c|c|c|c|}
\hline $\mathrm{H}$ & -8.455000 & 1.567000 & -3.344000 \\
\hline $\mathrm{H}$ & -6.752000 & 3.717000 & -4.746000 \\
\hline $\mathrm{H}$ & -7.505000 & 3.885000 & -3.149000 \\
\hline $\mathrm{H}$ & -8.515000 & 3.741000 & -4.598000 \\
\hline $\mathrm{H}$ & 6.414000 & 2.229000 & -5.114000 \\
\hline $\mathrm{H}$ & 6.461000 & 0.587000 & -5.783000 \\
\hline $\mathrm{H}$ & 5.528000 & 1.848000 & -6.591000 \\
\hline $\mathrm{H}$ & 7.201000 & -4.262000 & -1.704000 \\
\hline $\mathrm{H}$ & 7.256000 & -2.516000 & -1.977000 \\
\hline $\mathrm{H}$ & 7.135000 & -3.154000 & -0.332000 \\
\hline $\mathrm{H}$ & 5.819000 & 5.913000 & -1.815000 \\
\hline $\mathrm{H}$ & 5.972000 & 6.140000 & -0.064000 \\
\hline $\mathrm{H}$ & 4.865000 & 7.152000 & -0.994000 \\
\hline $\mathrm{H}$ & 6.596000 & 2.238000 & 5.017000 \\
\hline $\mathrm{H}$ & 6.937000 & 2.307000 & 3.288000 \\
\hline $\mathrm{H}$ & 6.907000 & 0.746000 & 4.132000 \\
\hline $\mathrm{H}$ & 4.569000 & -6.256000 & 4.703000 \\
\hline $\mathrm{H}$ & 4.599000 & -5.023000 & 5.973000 \\
\hline $\mathrm{H}$ & 5.818000 & -5.009000 & 4.695000 \\
\hline $\mathrm{H}$ & -3.834000 & 2.455000 & 4.030000 \\
\hline $\mathrm{H}$ & -5.427000 & 0.705000 & 4.966000 \\
\hline $\mathrm{H}$ & -4.052000 & -0.123000 & 5.694000 \\
\hline $\mathrm{N}$ & -4.057000 & -0.209000 & 3.622000 \\
\hline $\mathrm{C}$ & -4.439000 & -1.539000 & 3.511000 \\
\hline $\mathrm{H}$ & -4.057000 & 0.333000 & 2.757000 \\
\hline $\mathrm{O}$ & -4.768000 & -2.217000 & 4.483000 \\
\hline $\mathrm{N}$ & -4.343000 & -2.036000 & 2.224000 \\
\hline $\mathrm{C}$ & -4.982000 & -3.300000 & 1.875000 \\
\hline $\mathrm{H}$ & -4.278000 & -1.341000 & 1.483000 \\
\hline $\mathrm{C}$ & -6.425000 & -3.130000 & 1.376000 \\
\hline $\mathrm{H}$ & -4.963000 & -3.923000 & 2.773000 \\
\hline $\mathrm{H}$ & -4.371000 & -3.791000 & 1.110000 \\
\hline $\mathrm{H}$ & -7.006000 & -2.658000 & 2.179000 \\
\hline $\mathrm{C}$ & -7.108000 & -4.441000 & 0.950000 \\
\hline $\mathrm{H}$ & -6.428000 & -2.428000 & 0.529000 \\
\hline $\mathrm{H}$ & -8.168000 & -4.229000 & 0.753000 \\
\hline $\mathrm{H}$ & -7.094000 & -5.149000 & 1.791000 \\
\hline $\mathrm{C}$ & -6.497000 & -5.105000 & -0.292000 \\
\hline $\mathrm{H}$ & -6.475000 & -4.419000 & -1.148000 \\
\hline $\mathrm{H}$ & -7.078000 & -5.987000 & -0.586000 \\
\hline $\mathrm{H}$ & -5.469000 & -5.439000 & -0.111000 \\
\hline $\mathrm{C}$ & 5.392000 & 1.034000 & -0.179000 \\
\hline $\mathrm{C}$ & 4.554000 & -0.200000 & 0.168000 \\
\hline $\mathrm{N}$ & 3.181000 & 0.205000 & 0.633000 \\
\hline $\mathrm{H}$ & 4.422000 & -0.857000 & -0.692000 \\
\hline $\mathrm{H}$ & 4.995000 & -0.777000 & 0.982000 \\
\hline $\mathrm{H}$ & 2.558000 & -0.600000 & 0.869000 \\
\hline $\mathrm{H}$ & 2.693000 & 0.762000 & -0.077000 \\
\hline $\mathrm{H}$ & 3.224000 & 0.790000 & 1.478000 \\
\hline $\mathrm{H}$ & 4.912000 & 1.577000 & -1.001000 \\
\hline $\mathrm{H}$ & 5.407000 & 1.710000 & 0.685000 \\
\hline $\mathrm{C}$ & 6.809000 & 0.661000 & -0.568000 \\
\hline $\mathrm{C}$ & 9.440000 & -0.025000 & -1.289000 \\
\hline $\mathrm{C}$ & 7.768000 & 0.371000 & 0.414000 \\
\hline $\mathrm{C}$ & 7.186000 & 0.598000 & -1.915000 \\
\hline $\mathrm{C}$ & 8.493000 & 0.260000 & -2.274000 \\
\hline $\mathrm{C}$ & 9.072000 & 0.029000 & 0.058000 \\
\hline $\mathrm{H}$ & 7.495000 & 0.425000 & 1.466000 \\
\hline $\mathrm{H}$ & 6.451000 & 0.820000 & -2.684000 \\
\hline $\mathrm{H}$ & 8.771000 & 0.227000 & -3.324000 \\
\hline $\mathrm{H}$ & 9.804000 & -0.187000 & 0.831000 \\
\hline $\mathrm{H}$ & 10.458000 & -0.284000 & -1.566000 \\
\hline $\mathrm{Cl}$ & -3.743000 & 0.848000 & 0.408000 \\
\hline
\end{tabular}

$\begin{array}{llll}\mathrm{H} & -8.455000 & 1.567000 & -3.344000\end{array}$

$\begin{array}{lll}-7.505000 & 3.885000 & -3.149000\end{array}$

$\begin{array}{lll}-8.515000 & 3.741000 & -4.598000\end{array}$

$\begin{array}{lll}6.461000 & 0.587000 & -5.783000\end{array}$

$\begin{array}{lll}5.528000 & 1.848000 & -6.591000\end{array}$

$\begin{array}{lll}7.256000 & -2.516000 & -1.977000\end{array}$

$\begin{array}{lll}7.135000 & -3.154000 & -0.332000\end{array}$

$-5.913000-1.815000$

$\begin{array}{lll}4.865000 & 7.152000 & -0.994000\end{array}$

$\begin{array}{lll}6.596000 & 2.238000 & 5.017000\end{array}$

$\begin{array}{lll}6.907000 & 0.746000 & 4.132000\end{array}$

$\begin{array}{lll}4.569000 & -6.256000 & 4.703000\end{array}$

$\begin{array}{lll}5.818000 & -5.009000 & 4.695000\end{array}$

$\begin{array}{lll}3.834000 & 2.455000 & 4.030000\end{array}$

$0.705000 \quad 4.966000$

$\begin{array}{lll}-4.057000 & -0.209000 & 3.622000\end{array}$

$\begin{array}{lll}-4.439000 & -1.539000 & 3.511000\end{array}$

$\begin{array}{lll}-4.768000 & -2.217000 & 4.483000\end{array}$

$\begin{array}{lll}-4.343000 & -2.036000 & 2.224000\end{array}$

$\begin{array}{lll}-4.278000 & -1.341000 & 1.483000\end{array}$

$\begin{array}{lll}-6.425000 & -3.130000 & 1.376000\end{array}$

$-4.371000-3.791000$

$\begin{array}{lll}-7.006000 & -2.658000 & 2.179000\end{array}$

$\begin{array}{lll}-7.108000 & -4.441000 & 0.950000\end{array}$

$\begin{array}{lll}-8.168000 & -4.229000 & 0.753000\end{array}$

$-7.094000 \quad-5.149000 \quad 1.791000$

$\begin{array}{lll}-6.475000 & -4.419000 & -1.148000\end{array}$

$-5.987000-0.586000$

$5.392000-1.034000-0.179000$

$4.554000 \quad-0.200000 \quad 0.168000$

$\begin{array}{lll}0.205000 & 0.633000\end{array}$

$\begin{array}{lll}4.995000 & -0.777000 & 0.982000\end{array}$

$\begin{array}{ll}-0.600000 & 0.869000\end{array}$

$0.762000-0.077000$

$1.577000-1.001000$

$1.710000 \quad 0.685000$

$-0.661000-0.568000$

$\begin{array}{llll}\text { C } & -7.593000 & 3.387000 & -4.122000\end{array}$ 